\title{
Crack tip asymptotic field and K- dominant region for anisotropic semi-circular bend specimen
}

\section{Journal Article}

\section{Author(s):}

Nejati, Morteza; Ghouli, Saeid; Ayatollahi, Majid R.

Publication date:

2020-10

Permanent link:

https://doi.org/10.3929/ethz-b-000428107

\section{Rights / license:}

Creative Commons Attribution-NonCommercial-NoDerivatives 4.0 International

\section{Originally published in:}

Theoretical and Applied Fracture Mechanics 109, https://doi.org/10.1016/j.tafmec.2020.102640 


\title{
Crack tip asymptotic field and K-dominant region for anisotropic semi- circular bend specimen
}

\author{
Morteza Nejati $^{\mathrm{a}, *}$, Saeid Ghouli ${ }^{\mathrm{b}}$, Majid R. Ayatollahi ${ }^{\mathrm{b}}$ \\ ${ }^{a}$ Department of Earth Sciences, ETH Zurich, Switzerland \\ ${ }^{\mathrm{b}}$ Fatigue and Fracture Research Laboratory, School of Mechanical Engineering, Iran University of Science and Technology, Tehran, Iran
}

\section{A R T I C L E I N F O}

\section{Keywords:}

Semi-circular bend (SCB)

Anisotropy

Finite element

T-stress

Higher order parameters

K-dominant

\begin{abstract}
A B S T R A C T
This study reports the coefficients of the crack tip asymptotic field for the semi-circular bend (SCB) specimen made of anisotropic rocks when subjected to pure mode I loading. The finite element over-deterministic method is employed to determine the singular and higher order terms of the crack tip asymptotic field for a wide range of geometry and anisotropy parameters associated with the Mode SCB test. These parameters are helpful when analysing the fracture path and failure mechanism of anisotropic rocks in cases where the process zone is large compared to the crack length. We also apply an energy-based criterion on the crack tip fields to evaluate the size of the K-dominant region as a function of geometry configuration and anisotropy. It is concluded that the ISRMsuggested size requirement for the SCB samples of rock can yield underestimated values of fracture toughness due to the constraints applied to the FPZ development.
\end{abstract}

\section{Introduction}

Fracture mechanics of rock-type materials has invariably been an important topic in many industrial applications including geo-energy utilization systems such as enhanced geothermal reservoirs, civil construction projects such as tunnelling, petroleum engineering operations such as hydro-fracturing, as well as mining and underground excavations. In all these fields, rock mass is subjected to a complex mechanical, thermal and/or hydraulic loads that can cause existing fractures to propagate, or new fractures to be created. In view of the fact that rock materials often manifest quasi-brittle behaviour, the fracturing process is rather instantaneous, which can potentially lead to catastrophic and unforeseen circumstances. Moreover, many rock materials including granite, schist, sandstone, shale, limestone, etc. exhibit different extents of anisotropy (in elasticity, strength and fracture toughness), that makes it vital to correctly evaluate the influence of anisotropy on fracture growth, whereby providing insight into a better characterisation of rock masses.

A frequent type of rock failure in engineering applications occurs due to the mode I loading condition, hydro-fracturing being an important example. In such cases, the intensity of the near-tip crack fields is mostly controlled by the mode I stress intensity factor (SIF), $K_{\mathrm{I}}$. According to the linear elastic fracture mechanics (LEFM) theory, the onset of fracture growth occurs at a critical load where the SIF reaches the fracture toughness of the rock material $\left(K_{\mathrm{I}}=K_{\mathrm{Ic}}\right)$. Among existing tests available to measure mode I fracture toughness of rocks, the semicircular bend (SCB) test is a preferable choice owing to its favourable properties of the test, that are low amount of machining and material required per specimen, and the simple loading configuration [1]. This is the reason why the SCB test has been widely employed for fracture toughness measurement of isotropic [2-8] as well as anisotropic [9-16] rocks.

Most past works on the determination of SCB crack parameters have focused on the computation of the first two terms only (the SIF and Tstress), targeting mainly isotropic materials $[17,2,18,1]$. Only recently was the SCB test modified for anisotropic rocks, using an asymmetric loading setup [19]. This was followed by the computation of the SIFs for a wide range of several geometrical and anisotropy factors to conveniently measure the fracture toughness. Recent tests on anisotropic granitic and shale samples have demonstrated that along with the SIFs, the higher order coefficients are also important test parameters since they have a significant impact on the fracture growth $[16,20]$. This is because rock materials often possess large fracture process zones (FPZs) that normally stay out of the K-dominant region for small samples [21-23,15]. This makes it crucial to consider the higher order terms of the crack tip fields for growth analyses, which requires the accurate calculation of these parameters as an initial step. Note that the higher order parameters have been reported for various Mode I cracked

\footnotetext{
* Corresponding author.

E-mail address: mnejati@ethz.ch (M. Nejati).
} 


\author{
Nomenclature \\ a Crack length \\ $A_{n}, B_{n}, A_{n}^{*}, B_{n}^{*}, n=1,3,4, \ldots$ Coefficients of modes I and II terms \\ and their normalised forms \\ $A_{2}, B_{2} \quad$ Constituents of the T-stress and the rigid body ro- \\ tation \\ $E_{1}, E_{2} \quad$ In-plane and transverse Young's moduli \\ $F \quad$ Applied load on the SCB specimen \\ $G_{12}, G_{12}^{s v} \quad$ Transverse shear modulus and its approximation by \\ Saint-Venant \\ $i \quad$ Imaginary unit number \\ $K_{\mathrm{I}}, K_{\mathrm{II}} \quad$ Modes I and II stress intensity factors \\ $K_{\text {Ic }} \quad$ Mode I fracture toughness \\ $P, P_{j}, j=1,2$ Original and transformed plane problems related by \\ an affine transformation \\ $p_{j}, q_{j}, j=1,2$ Complex constants in deformation field \\ $R, t \quad$ Radius and thickness of the SCB specimen \\ $r, \theta \quad$ Polar coordinates of a point near the crack tip \\ $r_{K}, L_{\mathrm{FPZ}} \quad$ Size of K-dominant region and fracture process zone \\ $\boldsymbol{S}, S_{i j}, i, j=1,2,6$ Plane stress compliance matrix and its $i j$ com- \\ ponent in the off-axis coordinate system $x y$ ro- \\ tated by an angle $-\beta$ from $x^{\prime} y^{\prime}$ \\ $\overline{\boldsymbol{S}}, \bar{S}_{i j}, i, j=1,2,6$ Plane strain compliance matrix and its $i j$ com- \\ ponent in the off-axis coordinate system $x y$ ro- \\ tated by an angle $-\beta$ from $x^{\prime} y^{\prime}$ \\ $\boldsymbol{S}^{\prime}, S_{i j}^{\prime}, i, j=1,2,6$ Plane stress compliance matrix and its $i j$ com- \\ ponent in the material coordinate system $x^{\prime} y^{\prime}$ \\ $S_{1}, S_{2}$ \\ Span lengths of bottom supports for SCB specimen \\ under asymmetric three-point bend loading
}

$T, T^{*} \quad$ T-stress and its normalised form

$u, v \quad$ Displacement components along $x$ and $y$ directions

$u_{0}, v_{0} \quad$ Rigid body translations of the crack tip along $x$ and $y$ axes

$x, y, x_{j}, y_{j}, j=1,2$ Cartesian coordinates in $P$ and $P_{j}$ planes

$z, z_{j}, j=1,2$ Complex variables in $P$ and $P_{j}$ planes

$z_{0} \quad$ Crack tip location

$\beta \quad$ Angle between the anisotropy orientation and the horizontal plane, or crack plane in cracked bodies

$\mu_{j}, \bar{\mu}_{j}, j=1,2$ Conjugate pair of the roots of the characteristic equation in the off-axis coordinate system $x y$, known as complex parameters

$\mu_{j}^{\prime}, \bar{\mu}_{j}^{\prime}, j=1,2$ Complex parameters in the material coordinate system $x^{\prime} y^{\prime}$

$v_{21} \quad$ Transverse Poisson's ratio

$\omega \quad$ Rigid body rotation of the crack with respect to the crack tip

$\phi \quad$ Airy stress function

$\mathfrak{R}, \mathfrak{I} \quad$ Operators which output the real and imaginary parts of the enclosed argument

$\rho_{j}, \delta_{j}, j=1,2$ Real and imaginary parts of $\mu_{j}$

$\sigma, \varepsilon \quad$ Stress and strain vectors in the off-axis (crack) coordinate system $x y$

$\sigma_{t} \quad$ Tensile strength

$\sigma_{x}, \sigma_{y}, \tau_{x y} \quad$ In-plane normal and shear stress components in the off-axis (crack) coordinate system $x y$

$W \quad$ Strain energy

$\xi, \eta \quad$ Anisotropy ratios of the Young's modulus and the apparent shear modulus specimens of isotropic materials, including the standard compact tension specimen [24], the notched three-point bend beam $[25,26]$ and the wedge splitting specimen [27]. These values have then been employed to investigate the deterministic size effect on the strength of cracked concrete structures $[28,29]$. Similarly, the higher order terms of the SCB test for both isotropic [4,30] and anisotropic materials can be of great use in evaluating the size effect on fracture growth. However, the literature lacks these data for anisotropic SCB specimens.

This paper uses the finite element over-deterministic method (FEOD), recently developed by the authors [31], to determine the coefficients of the first five terms of mode I asymptotic crack tip field for the SCB specimen, for a wide range of geometrical and material factors. We then characterise the K-dominant region in the SCB specimen of an anisotropic rock. This is done by evaluating the strain energy over a circular area around the crack tip. It is shown that the size of K-dominant zone depends on the geometry, loading as well as the anisotropy parameters. We also discuss the implication of the FPZ developing outside the K-dominant region, that is a small SCB specimen can cause the FPZ not to fully develop, leading to the underestimation of the fracture toughness. It is manifested that in order to obtain reliable values of the fracture toughness, the specimen size (i.e. disk radius) should be sufficiently large so that the FPZ fits inside the K-zone.

\section{Theoretical background}

Consider a plane deformation problem in an anisotropic medium where the compatibility condition is formulated in terms of the Airy stress function $\phi$ as [32]
$S_{22} \frac{\partial^{4} \phi}{\partial x^{4}}-2 S_{26} \frac{\partial^{4} \phi}{\partial x^{3} \partial y}+\left(2 S_{12}+S_{66}\right) \frac{\partial^{4} \phi}{\partial x^{2} \partial y^{2}}-2 S_{16} \frac{\partial^{4} \phi}{\partial x \partial y^{3}}+S_{11} \frac{\partial^{4} \phi}{\partial y^{4}}=0$

where $S_{i j}, i, j=1,2,6$ are the constituents of the plane stress compliance matrix $\boldsymbol{S}$ of the anisotropic material, and $x$ and $y$ are the components of the Cartesian coordinate system. For the plane strain condition, it is sufficient to replace $S_{i j}$ with $\bar{S}_{i j}=S_{i j}-S_{i 3} S_{j 3} / S_{33}$ to form the plane strain compliance matrix $\bar{S}$. The Airy stress function, $\phi$, ensures the satisfaction of the equilibrium condition, using the following definition of the stress components:

$\sigma_{x}=\frac{\partial^{2} \phi}{\partial y^{2}}, \quad \sigma_{y}=\frac{\partial^{2} \phi}{\partial x^{2}}, \tau_{x y}=-\frac{\partial^{2} \phi}{\partial x \partial y}$.

Utilising the form $\phi=\phi(z)$ in Eq. (1) yields the characteristic equation as

$S_{11} \mu^{4}-2 S_{16} \mu^{3}+\left(2 S_{12}+S_{66}\right) \mu^{2}-2 S_{26} \mu+S_{22}=0$,

with $z=x+\mu y$ being a complex variable. The roots of the characteristic equation, called complex parameters, occur in complex conjugate pairs: $\mu_{1}=\rho_{1}+i \delta_{1}, \mu_{2}=\rho_{2}+i \delta_{2}, \mu_{3}=\bar{\mu}_{1}$ and $\mu_{4}=\bar{\mu}_{2}$. The complex parameters uniquely quantify how severely an anisotropic solution may deviate from an isotropic one for which $\mu_{1}=\mu_{2}=i$.

We herein consider the case where the plane deformation problem is parallel to a symmetry plane of the orthotropic material. In this case, there are two principal axes $x^{\prime}$ and within the plane, with respect to which the planar deformation is symmetrical (see Fig. 1). With reference to these principal axes, only four independent material constants are required to characterise the equations in the plane stress 


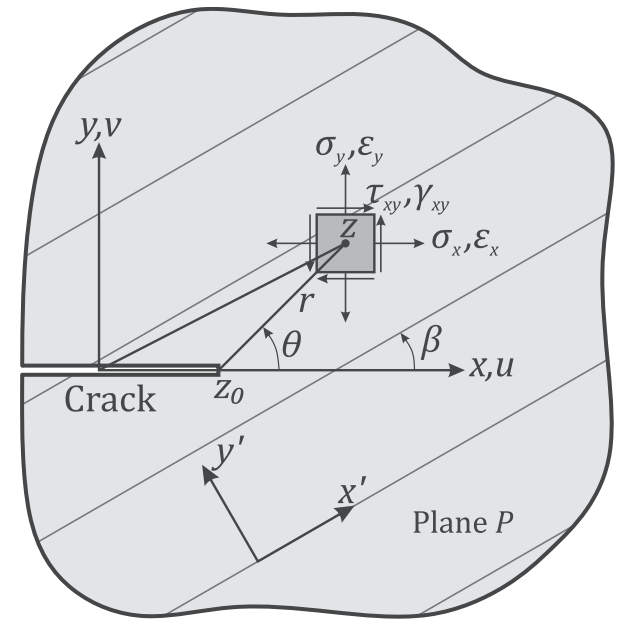

Fig. 1. A cracked anisotropic plane with the material orientation forming angle $\beta$ with respect to the crack.
Implementing these two physical parameters in Eq. (4) gives

$\mu^{\prime 4}+\left(\frac{1+\xi}{\eta}+2 \xi \nu_{21} \frac{1-\eta}{\eta}\right) \mu^{\prime 2}+\xi=0$.

Once Eq. (5) is solved to evaluate $\mu_{1}^{\prime}$ and $\mu_{2}^{\prime}$ in $x^{\prime} y^{\prime}$ coordinate system, the complex parameters $\mu_{1}$ and $\mu_{2}$ associated with the crack coordinate system $x y$ are obtained as [32]

$\mu_{j}=\frac{\mu_{j}^{\prime} \cos \beta+\sin \beta}{\cos \beta-\mu_{j}^{\prime} \sin \beta}, \quad(j=1,2)$,

in which $\beta$ is the angle between the material orientation and the crack (see Fig. 1). The characteristic equation normally returns distinct complex roots $\left(\mu_{1} \neq \mu_{2}\right)$ in general anisotropic problems.

Ghouli et al. [35] put forward a stress-based definition for the anisotropic fracture modes based on the criterion that at $\theta=0^{\circ}, \tau_{x y}=0$ for pure mode I, and $\sigma_{y}=0$ for pure mode II, whereby re-visiting the stress and displacement fields as infinite series expansions, respectively in Eqs. (7) and (8):

$$
\begin{aligned}
& {\left[\begin{array}{c}
\sigma_{x} \\
\sigma_{y} \\
\tau_{x y}
\end{array}\right]=2 \Re\left(\sum_{n=1}^{\infty} A_{n} \frac{i(n+1)^{2}}{\mu_{1}-\mu_{2}} r^{\frac{n}{2}-1}\left[\begin{array}{c}
\mu_{2}^{2} \mu_{1}^{\frac{(-1)^{n+1}+1}{2}}\left(\cos \theta+\mu_{2} \sin \theta\right)^{\frac{n}{2}-1}-\mu_{1}^{2} \mu_{2}^{\frac{(-1)^{n+1}+1}{2}}\left(\cos \theta+\mu_{1} \sin \theta\right)^{\frac{n}{2}-1} \\
\mu_{1}^{\frac{(-1)^{n+1}+1}{2}}\left(\cos \theta+\mu_{2} \sin \theta\right)^{\frac{n}{2}-1}-\mu_{2} \frac{(-1)^{n+1}+1}{2}\left(\cos \theta+\mu_{1} \sin \theta\right)^{\frac{n}{2}-1} \\
-\left(\mu_{2} \mu^{\frac{(-1)^{n+1}+1}{2}}\left(\cos \theta+\mu_{2} \sin \theta\right)^{\frac{n}{2}-1}-\mu_{1} \mu_{2}^{\frac{(-1)^{n+1}+1}{2}}\left(\cos \theta+\mu_{1} \sin \theta\right)^{\frac{n}{2}-1}\right)
\end{array}\right]\right)} \\
& +2 \Re\left(\sum_{n=1}^{\infty} B_{n} \frac{i^{(n+1)^{2}}}{\mu_{1}-\mu_{2}} r^{\frac{n}{2}-1}\left[\begin{array}{c}
\mu_{2}^{2} \mu_{1}^{\frac{(-1)^{n}+1}{2}}\left(\cos \theta+\mu_{2} \sin \theta\right)^{\frac{n}{2}-1}-\mu_{1}^{2} \mu_{2}^{\frac{(-1)^{n}+1}{2}}\left(\cos \theta+\mu_{1} \sin \theta\right)^{\frac{n}{2}-1} \\
\mu_{1}^{\frac{(-1)^{n}+1}{2}}\left(\cos \theta+\mu_{2} \sin \theta\right)^{\frac{n}{2}-1}-\mu_{2}^{\frac{(-1)^{n}+1}{2}}\left(\cos \theta+\mu_{1} \sin \theta\right)^{\frac{n}{2}-1} \\
-\left(\mu_{2} \mu_{1}^{\frac{(-1)^{n}+1}{2}}\left(\cos \theta+\mu_{2} \sin \theta\right)^{\frac{n}{2}-1}-\mu_{1} \mu_{2}^{\frac{(-1)^{n}+1}{2}}\left(\cos \theta+\mu_{1} \sin \theta\right)^{\frac{n}{2}-1}\right.
\end{array}\right)\right),
\end{aligned}
$$

$$
\begin{aligned}
& {\left[\begin{array}{l}
u \\
v
\end{array}\right]=\left[\begin{array}{l}
u_{0} \\
v_{0}
\end{array}\right]+4 \Re\left(\sum_{n=1}^{\infty} A_{n} \frac{i(n+1)^{2}}{\mu_{1}-\mu_{2}} \frac{1}{n} r^{\frac{n}{2}}\left[\begin{array}{l}
p_{2} \mu_{1}^{\frac{(-1)^{n+1}+1}{2}}\left(\cos \theta+\mu_{2} \sin \theta\right)^{\frac{n}{2}}-p_{1} \mu_{2}^{\frac{(-1)^{n+1}+1}{2}}\left(\cos \theta+\mu_{1} \sin \theta\right)^{\frac{n}{2}} \\
q_{2} \mu_{1}^{\frac{(-1)^{n+1}+1}{2}}\left(\cos \theta+\mu_{2} \sin \theta\right)^{\frac{n}{2}}-q_{1} \mu_{2}^{\frac{(-1)^{n+1}+1}{2}}\left(\cos \theta+\mu_{1} \sin \theta\right)^{\frac{n}{2}}
\end{array}\right]\right)} \\
& +4 \Re\left(\sum_{n=1}^{\infty} B_{n} \frac{i(n+1)^{2}}{\mu_{1}-\mu_{2}} \frac{1}{n} r^{\frac{n}{2}}\left[\begin{array}{l}
p_{2} \mu_{1}^{\frac{(-1)^{n}+1}{2}}\left(\cos \theta+\mu_{2} \sin \theta\right)^{\frac{n}{2}}-p_{1} \mu_{2} \frac{(-1)^{n}+1}{2}\left(\cos \theta+\mu_{1} \sin \theta\right)^{\frac{n}{2}} \\
q_{2} \mu_{1}^{\frac{(-1)^{n}+1}{2}}\left(\cos \theta+\mu_{2} \sin \theta\right)^{\frac{n}{2}}-q_{1} \mu_{2}^{\frac{(-1)^{n}+1}{2}}\left(\cos \theta+\mu_{1} \sin \theta\right)^{\frac{n}{2}}
\end{array}\right]\right) .
\end{aligned}
$$

condition: Young's moduli $E_{1}$ and $E_{2}$ along the principal axes $x^{\prime}$ and $y^{\prime}$, the shear modulus $G_{12}$ and the Poisson's ratio $\nu_{21}$. The compliance matrix associated with this principal coordinate system, $\boldsymbol{S}^{\prime}$, incorporates elements $S_{11}^{\prime}=1 / E_{1}, \quad S_{22}^{\prime}=1 / E_{2}, \quad S_{12}^{\prime}=-v_{21} / E_{2}, \quad S_{66}^{\prime}=1 / G_{12} \quad$ and $S_{16}^{\prime}=S_{26}^{\prime}=0$, yielding a simplified characteristic equation:

$\mu^{\prime 4}+\left(\frac{E_{1}}{G_{12}}-2 \nu_{21} \frac{E_{1}}{E_{2}}\right) \mu^{\prime 2}+\frac{E_{1}}{E_{2}}=0$

The four constants $E_{1}, E_{2}, \nu_{21}$ and $G_{12}$ are independent parameters, albeit Saint Venant [33] proposed an approximation of the transverse shear modulus in terms of the other elastic constants: $1 / G_{12}^{s v}=1 / E_{1}+1 / E_{2}+2 \nu_{21} / E_{2}$. Making use of this estimate, Nejati et al. [19] and Aminzadeh et al. [34] introduced the two non-dimensional parameters $\xi=E_{1} / E_{2}$ and $\eta=G_{12} / G_{12}^{s v}$ as the anisotropy ratios of the Young's modulus and the apparent shear modulus. The greater the deviation of $\xi$ and $\eta$ from unity, the more significant the directional dependency of the Young's modulus and the shear modulus.
Here, $A_{n}$ and $B_{n}$ represent the coefficients of mode I and II, respectively. These parameters must be real numbers so as to satisfy the traction-free boundary conditions of the crack faces. The coefficients of the first terms relate to the SIFs through $K_{\mathrm{I}}=2 \sqrt{2 \pi} A_{1}$ and $K_{\mathrm{II}}=2 \sqrt{2 \pi} B_{1}$. The integration constants $u_{0}$ and $v_{0}$ in the displacement field are the rigid body translation of the crack tip in the directions $x$ and $y$, respectively. The parameters $p_{j}$ and $q_{j}$ are complex numbers defined as functions of compliance matrix elements: $p_{j}=S_{11} \mu_{j}^{2}-S_{16} \mu_{j}+S_{12}$ and $q_{j}=S_{12} \mu_{j}-S_{26}+S_{22} / \mu_{j}$. Note that since Eqs. (7) and (8) represent the crack fields in the crack tip coordinate system, complex parameters $\mu_{j}$ are also to be determined in the same system of coordinates. Evaluated from Eqs. (7) and (8), the coefficients of the second terms, $A_{2}$ and $B_{2}$, construct two physical parameters, $T$ and $\omega$ [35]:

$T=2 \Im\left[A_{2}\left(\mu_{1}+\mu_{2}\right)+B_{2} \mu_{1} \mu_{2}\right]$,

$\omega=2 S_{11} \Im\left[\bar{\mu}_{1} \bar{\mu}_{2}\left(A_{2}+B_{2}\left(\mu_{1}+\mu_{2}\right)\right)\right]$,

where $\mathfrak{I}$ designates the imaginary part of the enclosed complex 
argument. $T$ is a constant stress term, known as the T-stress, acting parallel to the crack flanks, and $\omega$ is the rigid body rotation of the cracked body with respect to the crack tip. It is worth mentioning that all $S_{i j}$ constants are superseded by $\bar{S}_{i j}$ when dealing with a plane strain problem.

\section{Crack tip parameters of the SCB specimen}

Semi-circular bend (SCB) specimen is one of the suggested methods by the International Society for Rock Mechanics (ISRM) for the measurement of the mode I static fracture toughness of rock materials [1]. The SCB test is based on the three-point bend loading configuration, where a compressive load yields a tension-induced fracture growth (see Fig. 2a). The SCB test is popular due to the following meritorious points: (a) small amount of core material; (b) relatively simple machining process; (c) suitability for both isotropic and anisotropic rocks. These traits have made the SCB specimen a good candidate for experimental measurements of fracture properties, particularly the mode I fracture toughness. Note that we use the definition proposed by Ayatollahi and Zakeri [36] for applying modes I and II crack loading. In line with this definition, mode I crack loading occurs when $K_{\mathrm{II}}=0$ (i.e. $B_{1}=0$ ), while mode II loading is applied when $K_{\mathrm{I}}=0$ (i.e. $A_{1}=0$ ). Unlike the classical definition of the crack loading, this description does not impose any restriction on the higher order parameters.

In order to apply mode I loading to the SCB specimen, a vertical precrack, that is collinear with the load, must be machined at the centre of the test sample. When the rock is isotropic, the roller supports are placed in symmetrical spans $S_{1}=S_{2}$ (refer to Bahrami et al. [8,37] and Sedighi et al. [38] for the necessity of the usage of free-rolling supports in the SCB tests). However, for SCB specimens made of anisotropic rocks that have anisotropy orientation forming an angle $\beta \neq 0^{\circ}, 90^{\circ}$, asymmetrical support spans are required to enforce mode I loading [19] (see Fig. 2a). In order to normalise the crack parameters of the SCB specimen, we use the following relations:

$$
\begin{aligned}
A_{n} & =A_{n}^{*} \frac{F}{2 R t} a^{1-\frac{n}{2}}, \quad B_{n}=B_{n}^{*} \frac{F}{2 R t} a^{1-\frac{n}{2}}, \quad(n=1,3,4, \ldots) \\
T & =T^{*} \frac{F}{2 R t} .
\end{aligned}
$$

Here ${ }^{*}$ denotes the normalised crack parameter, $F$ is the applied compressive load, $R$ and $t$ represent the radius and the thickness of the SCB specimen, and $a$ is the length of the pre-cut crack. In this formulation, $A_{1}^{*}$ and $B_{1}^{*}$ are related to the geometry factors reported in Nejati et al. [19] as $Y_{\mathrm{I}}=2 \sqrt{2} A_{1}^{*}$ and $Y_{\mathrm{II}}=2 \sqrt{2} B_{1}^{*}$.
Table 1

Geometrical and material properties used for the calculation of crack parameters.

\begin{tabular}{llllll}
\hline$a / R$ & $S_{1} / R$ & $\xi=E_{1} / E_{2}$ & $\eta=G_{12} / G_{12}^{s v}$ & $\nu_{21}$ & $\beta\left({ }^{\circ}\right)$ \\
\hline $0.4,0.5,0.6$ & $0.6,0.8$ & $1,2,3,4$ & $1,0.7,1.3$ & 0.1 & $0,30,60,90$ \\
\hline
\end{tabular}

We employed the FEOD method to determine the crack parameters for the SCB test. The basic principle of this method is the usage of a large number of FE data points in order to calculate the crack tip parameters. This is conducted by forming an algebraic system of equations where the number of equations is more than the number of unknowns. In such case, we encounter an over-determined system of equations, with the solution given in a least squares sense. Solving such over-determined system yields a more precise calculation of the unknowns compared to the alternative determined system. We employed the finite element package Abaqus in order to model the SCB specimen, where eight-noded plane stress quadratic quadrilateral elements of type CPS8 with unit thickness discretise the solution domain. Fig. 2b shows a typical finite element model employed for analysing the current problem. The mid-side nodes at the crack tip elements are moved to the one-quarter position in order to capture the LEFM square root stress singularity of type $1 / \sqrt{r}$ at the crack tip $[39,40]$. The mesh size in the close vicinity of the crack tip, $l$, normalized by the crack length, $a$, reads $l / a=0.008$.

We determined the crack parameters for a wide range of crack length ratios, span ratios and material properties, as listed in Table 1. We point out that the elasticity solution of the crack problem hardly depends on the Poisson's ratio $\nu_{21}$ when $\eta$ is close to unity [19]. We therefore use an arbitrary value of $\nu_{21}=0.1$ to determine the crack parameters. For each geometry and material configuration, the ratio $S_{2} / S_{1}$ was determined in such a way that pure mode I crack loading is applied, i.e. $K_{\mathrm{II}}=B_{1}=0$. Here, we benefited from the values of $S_{2} / S_{1}$ ratio reported by Nejati et al. [19]. After performing the FE analyses, the over-deterministic method was employed to calculate the crack parameters for the SCB specimen.

In all the FEOD analyses, displacements are extracted from the FE nodes along a circular path centred at the crack tip. We used a truncated series expansion with forty terms (twenty mode I and twenty mode II) to obtain the crack tip parameters. However, we only report the coefficients of the first five terms for each mode. The detail on how

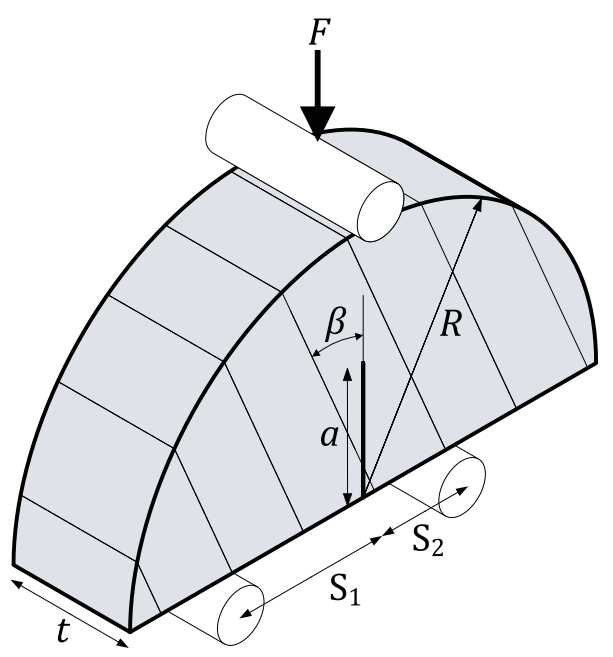

(a)

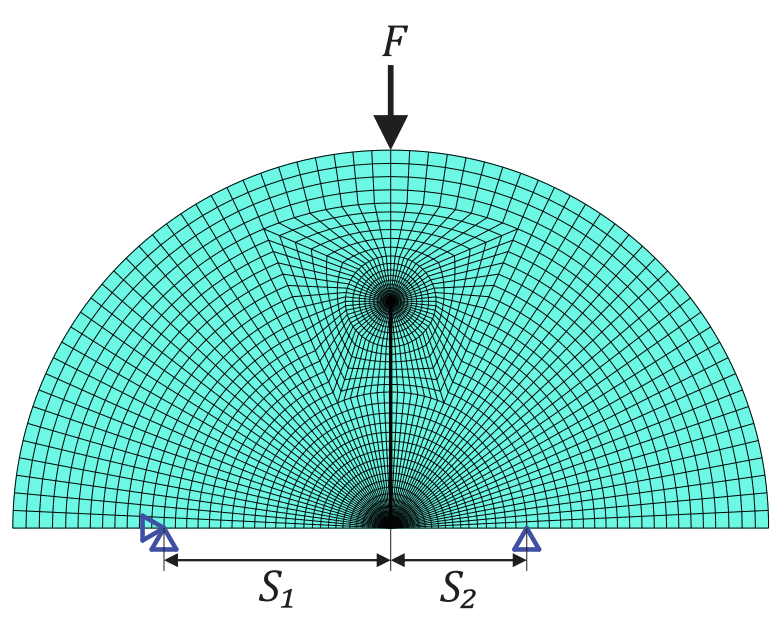

(b)

Fig. 2. (a) Semi-circular bend (SCB) test configuration with asymmetric loading; (b) Typical FE mesh used for the numerical modelling of the SCB specimen. 
Table 2

Normalised crack tip parameters $A_{n}^{*}, B_{n}^{*}(\mathrm{n}=1,3,4,5)$ and $T^{*}$ for the SCB specimen under mode I fracture: $a / R=0.4$ and $S_{1} / R=0.6$.

\begin{tabular}{|c|c|c|c|c|c|c|c|c|c|c|c|c|}
\hline$\eta$ & $\xi$ & $\beta\left({ }^{\circ}\right)$ & $S_{2} / S_{1}$ & $A_{1}^{*}$ & $B_{1}^{*}$ & $T^{*}$ & $A_{3}^{*}$ & $B_{3}^{*}$ & $A_{4}^{*}$ & $B_{4}^{*}$ & $A_{5}^{*}$ & $B_{5}^{*}$ \\
\hline \multirow[t]{13}{*}{1} & 1 & - & 1.00 & 1.3289 & 0.0000 & -0.5670 & -1.6729 & 0.0000 & 0.2956 & 0.0000 & -0.1494 & 0.0000 \\
\hline & \multirow[t]{4}{*}{2} & 0 & 1.00 & 1.3605 & 0.0000 & -0.6342 & -1.6621 & 0.0000 & -0.1755 & 0.0000 & -0.3274 & 0.0000 \\
\hline & & 30 & 0.60 & 0.9330 & 0.0000 & -0.8726 & -1.1508 & -0.4213 & -0.5914 & -0.1099 & 0.2147 & 0.3386 \\
\hline & & 60 & 0.68 & 0.9937 & 0.0000 & -0.8253 & -1.2348 & -0.3515 & -0.6128 & -0.0450 & 0.3122 & 0.3635 \\
\hline & & 90 & 1.00 & 1.2985 & 0.0000 & -0.5356 & -1.7080 & 0.0000 & -0.4021 & 0.0000 & 0.0570 & 0.0000 \\
\hline & \multirow[t]{4}{*}{3} & 0 & 1.00 & 1.3775 & 0.0000 & -0.6922 & -1.6617 & 0.0000 & -0.1106 & 0.0000 & -0.4053 & 0.0000 \\
\hline & & 30 & 0.48 & 0.7994 & 0.0000 & -0.9901 & -1.0062 & -0.5806 & -0.7575 & -0.1401 & 0.3783 & 0.6414 \\
\hline & & 60 & 0.62 & 0.9145 & 0.0000 & -0.8987 & -1.1564 & -0.4544 & -0.7104 & 0.0084 & 0.4926 & 0.6283 \\
\hline & & 90 & 1.00 & 1.2876 & 0.0000 & -0.5203 & -1.7657 & 0.0000 & -0.4394 & 0.0000 & 0.1599 & 0.0000 \\
\hline & \multirow[t]{4}{*}{4} & 0 & 1.00 & 1.3887 & 0.0000 & -0.7380 & -1.6644 & 0.0000 & -0.0675 & 0.0000 & -0.4500 & 0.0000 \\
\hline & & 30 & 0.43 & 0.7328 & 0.0000 & -1.0678 & -0.9399 & -0.6726 & -0.8790 & -0.1477 & 0.4916 & 0.9157 \\
\hline & & 60 & 0.59 & 0.8822 & -0.0001 & -0.9353 & -1.1388 & -0.5043 & -0.7394 & 0.0923 & 0.5916 & 0.8141 \\
\hline & & 90 & 1.00 & 1.2862 & 0.0000 & -0.5051 & -1.8421 & 0.0000 & -0.4501 & 0.0000 & 0.2164 & 0.0000 \\
\hline \multirow[t]{12}{*}{0.7} & \multirow[t]{4}{*}{2} & 0 & 1.00 & 1.3622 & 0.0000 & -0.7115 & -1.6623 & 0.0000 & -0.2099 & 0.0000 & -0.3006 & 0.0000 \\
\hline & & 30 & 0.57 & 0.8839 & -0.0001 & -0.8730 & -1.0933 & -0.4291 & -0.6789 & -0.1617 & 0.2976 & 0.3631 \\
\hline & & 60 & 0.76 & 1.0814 & 0.0001 & -0.7388 & -1.4054 & -0.3750 & -0.5803 & -0.0461 & 0.3530 & 0.4183 \\
\hline & & 90 & 1.00 & 1.3175 & 0.0000 & -0.5513 & -1.7595 & 0.0000 & -0.3727 & 0.0000 & -0.0023 & 0.0000 \\
\hline & \multirow[t]{4}{*}{3} & 0 & 1.00 & 1.3777 & 0.0000 & -0.7827 & -1.6625 & 0.0000 & -0.1583 & 0.0000 & -0.3679 & 0.0000 \\
\hline & & 30 & 0.48 & 0.7733 & 0.0000 & -0.9738 & -0.9832 & -0.6052 & -0.8965 & -0.2303 & 0.5139 & 0.7428 \\
\hline & & 60 & 0.68 & 0.9904 & -0.0001 & -0.8259 & -1.3167 & -0.4911 & -0.6864 & 0.0111 & 0.5840 & 0.6993 \\
\hline & & 90 & 1.00 & 1.3179 & 0.0000 & -0.5120 & -1.8682 & 0.0000 & -0.3924 & 0.0000 & 0.0729 & 0.0000 \\
\hline & \multirow[t]{4}{*}{4} & 0 & 1.00 & 1.3883 & 0.0000 & -0.8376 & -1.6662 & 0.0000 & -0.1235 & 0.0000 & -0.4078 & 0.0000 \\
\hline & & 30 & 0.43 & 0.7182 & 0.0000 & -1.0350 & -0.9401 & -0.7210 & -1.0787 & -0.2795 & 0.6978 & 1.1098 \\
\hline & & 60 & 0.65 & 0.9517 & 0.0000 & -0.8706 & -1.2946 & -0.5478 & -0.7095 & 0.1050 & 0.6957 & 0.8814 \\
\hline & & 90 & 1.00 & 1.3262 & 0.0000 & -0.4793 & -1.9942 & 0.0000 & -0.3949 & 0.0000 & 0.1206 & 0.0000 \\
\hline \multirow[t]{12}{*}{1.3} & \multirow[t]{4}{*}{2} & 0 & 1.00 & 1.3617 & 0.0000 & -0.5851 & -1.6735 & 0.0000 & 0.1499 & 0.0000 & -0.3442 & 0.0000 \\
\hline & & 30 & 0.62 & 0.9702 & -0.0001 & -0.8745 & -1.2073 & -0.4222 & 0.5452 & 0.0791 & 0.1761 & 0.3304 \\
\hline & & 60 & 0.64 & 0.9412 & 0.0000 & -0.8758 & -1.1474 & -0.3429 & 0.6332 & 0.0559 & 0.2978 & 0.3370 \\
\hline & & 90 & 1.00 & 1.2896 & 0.0000 & -0.5223 & -1.7000 & 0.0000 & 0.4260 & 0.0000 & 0.1104 & 0.0000 \\
\hline & \multirow[t]{4}{*}{3} & 0 & 1.00 & 1.3792 & 0.0000 & -0.6365 & -1.6707 & 0.0000 & 0.0758 & 0.0000 & -0.4278 & 0.0000 \\
\hline & & 30 & 0.49 & 0.8197 & -0.0001 & -1.0042 & -1.0389 & -0.5749 & 0.6842 & 0.0895 & 0.3096 & 0.5873 \\
\hline & & 60 & 0.58 & 0.8702 & 0.0000 & -0.9416 & -1.0788 & -0.4404 & 0.7324 & 0.0089 & 0.4576 & 0.6036 \\
\hline & & 90 & 1.00 & 1.2712 & 0.0000 & -0.5227 & -1.7290 & 0.0000 & 0.4796 & 0.0000 & 0.2461 & 0.0000 \\
\hline & \multirow[t]{4}{*}{4} & 0 & 1.00 & 1.3907 & 0.0000 & -0.6781 & -1.6716 & 0.0000 & 0.0269 & 0.0000 & -0.4747 & 0.0000 \\
\hline & & 30 & 0.43 & 0.7446 & 0.0001 & -1.0917 & -0.9589 & -0.6561 & 0.7729 & 0.0765 & 0.3835 & 0.8059 \\
\hline & & 60 & 0.56 & 0.8431 & 0.0000 & -0.9719 & -1.0680 & -0.4889 & 0.7693 & -0.0669 & 0.5549 & 0.7998 \\
\hline & & 90 & 1.00 & 1.2632 & 0.0000 & -0.5194 & -1.7751 & 0.0000 & 0.4990 & 0.0000 & 0.3210 & 0.0000 \\
\hline
\end{tabular}

to determine crack parameters from the FEOD method is given in detail in Ayatollahi et al. [31]. Table 2 presents the normalised crack parameters $A_{1}^{*}, B_{1}^{*}, T^{*}, A_{3}^{*} \sim A_{5}^{*}$ and $B_{3}^{*} \sim B_{5}^{*}$ for the case $a / R=0.4$ and $S_{1} / R=0.6$. We give comprehensive data on the normalised crack parameters and their corresponding $S_{2} / S_{1}$ for other ratios of $a / R$ and $S_{1} / R$ in Appendix A.

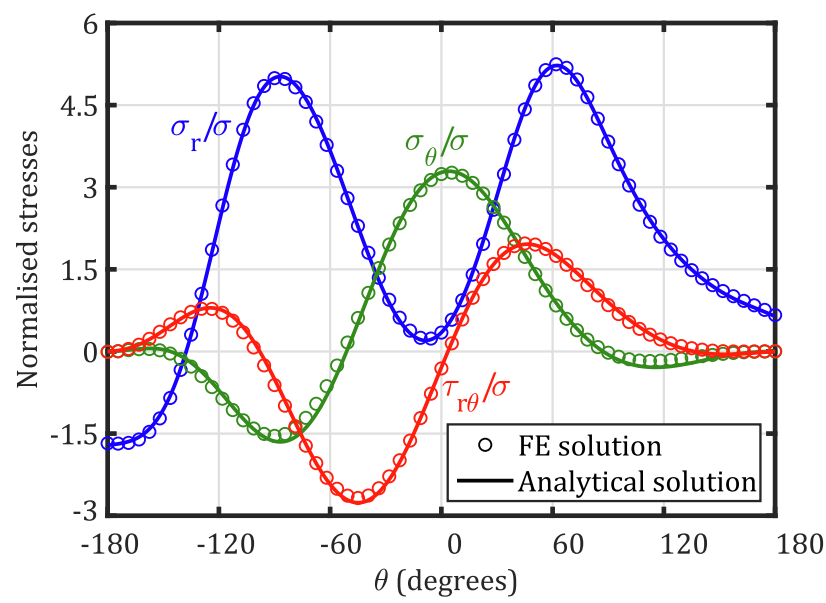

Fig. 3. Comparison between FE directly derived stresses and the analytical stress field in Eq. (7) assisted with the FEOD-calculated parameters. Stresses are normalised by $\sigma=F /(2 R t)$ and are plotted at a circular path with $r / a \approx 0.2$, for the case $a / R=0.4$ and $S_{1} / R=0.6$ with anisotropy parameters $\eta=1.3, \xi=4$ and $\beta=60^{\circ}$.
Fig. 3 verifies the veracity of the anisotropic stress field in Eq. (7) as well as the accuracy of the FEOD-calculated crack parameters. The first order parameters $A_{1}^{*}$ and $B_{1}^{*}$ (or equivalently $Y_{\mathrm{I}}$ and $Y_{\mathrm{II}}$ ) in Tables 2, A.1-A.5 also agree well with those reported by Nejati et al. [19]. It is seen that normalised T-stress, $T^{*}$, has mostly negative values, especially for the span ratio of $S_{1} / R=0.6$, that is perceived to compete against crack kinking under mode I condition. Note that crack may kink under mode I loading of anisotropic rocks, due to the directional dependency of the strength $[16,20]$. For $S_{1} / R=0.8$, on the other hand, the T-stress increases and often acquires positive values, accompanied by a subsequent higher chance of crack path instability and ensuing crack kinking. As demonstrated in Ayatollahi and Akbardoost [41] and Akbardoost and Ayatollahi [42], third order crack parameters, $A_{3}$ and $B_{3}$, may also highly influence the fracture behaviour of rock materials. According to our calculations, the normalised parameters $A_{3}^{*}$ and $B_{3}^{*}$ do not gain positive values in the SCB specimen subjected to mode I loading. Furthermore, $B_{3}^{*} \sim B_{5}^{*}$ vanish when beddings are collinear with or perpendicular to the crack plane (i.e. $\beta=0,90^{\circ}$ ), similar to the isotropic case. Benefiting from these reported higher order crack parameters, not only the analytical predictions of energy, stress, strain and displacement can be improved, but also the already-existing fracture criteria might be extended to account for other crack parameters besides the singular ones.

\section{K-dominant zone in the SCB specimen}

Major fracture growth models for both isotropic and anisotropic materials are formulated based on only the singular terms of crack tip 


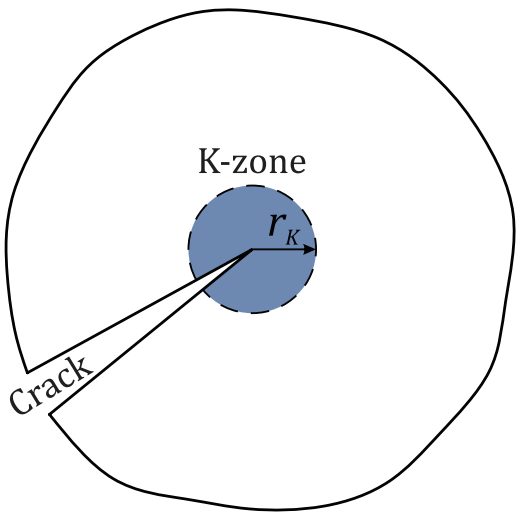

(a)

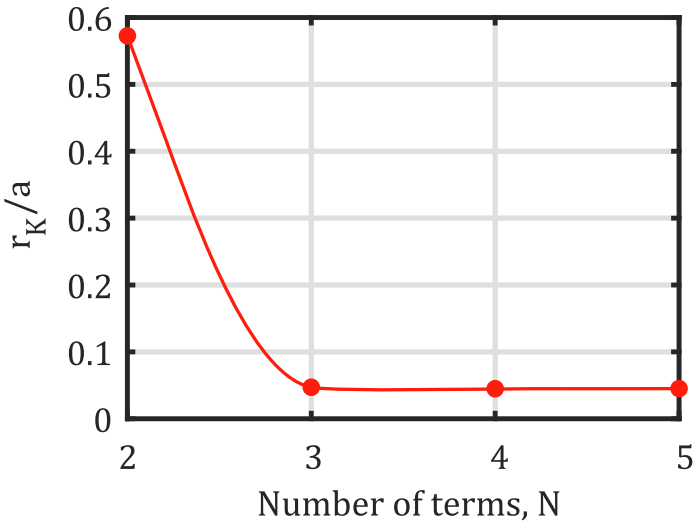

(b)

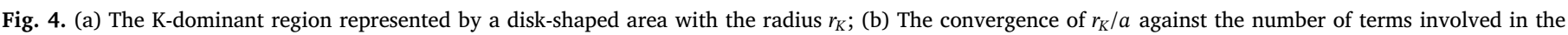
calculations of strain energy $\left(a / R=0.5, S_{1} / R=0.6, \eta=1, \xi=3, \beta=30^{\circ}\right)$.

fields [43-48]. These models assume that the FPZ develops in the close vicinity of the crack tip, where the singular terms are dominant and therefore sufficient to characterise crack tip fields. The crack tip region wherein the singular terms are sufficient to characterise stress is referred to as the $K$-dominant zone. This zone is often distinguished as a circular area with the radius of $r_{K}$ which depends on specimen size and geometry as well as the material anisotropy and the loading condition. For the evaluation of $r_{K}$, a stress-based method has often been employed [49-55]. This method determines $r_{K}$ as the distance from the crack tip along the crack plane $\left(\theta=0^{\circ}\right)$ at which the singular term of the stress component normal to the crack, $\sigma_{y}$, contributes to more than $90-95 \%$ of the total stress, indicating that the higher order parameters contribute only up to $5-10 \%$. Despite frequent use of this definition, this is not an ideal method to determine $r_{K}$ since it uses only one component of stress, $\sigma_{y}$, at one angle, $\theta=0^{\circ}$. In fact, the process of crack growth may involve the contribution of multiple components of the stress tensor. In addition, evaluating stress along $\theta=0^{\circ}$ may be irrelevant when crack kinking occurs, for example in mode I fracture growth of anisotropic rocks [16]. Note that the angle of fracture growth depends on the loading condition as well as the anisotropic properties.

Here, we introduce an energy-based approach to evaluate $r_{K}$. It relies on averaging the scalar quantity of the strain energy density, that includes the contribution of all components of stress and strain, over a circular region around the crack tip (see Fig. 4a). This definition scans a disk around the crack tip, and allows to consider all components of stress and strain and all possible angles to determine $r_{K}$. Let us define $r_{K}$ to be the radius of the region wherein the strain energy from the singular terms only is not more than $10 \%$ different from the strain energy obtained from infinite number of terms of the series solution i.e. the exact solution:

$\frac{\left|W_{\infty}-W_{1}\right|}{W_{\infty}}=0.1$

Here, the strain energy, $W$, is calculated within the disk-shaped area $A$ with a unit thickness, using the stress, $\sigma$, and strain, $\varepsilon$, vectors that are calculated based on the singular terms for $W_{1}$ and infinite number of terms for $W_{\infty}$ :

$W=\frac{1}{2} \int_{A} \sigma^{T} \varepsilon d A=\frac{1}{2} \int_{0}^{r_{K}} \int_{-\pi}^{\pi} \sigma^{T} \boldsymbol{S} \sigma d \theta r d r$,

Since the calculation of the exact strain energy $\left(W_{\infty}\right)$ requires the inclusion of infinite number of stress and strain terms, we suggest to truncate the series to a sufficient number in order to conveniently determine the strain energy. Fig. $4 \mathrm{~b}$ shows the variation of $r_{K}$ against the number of terms $(N)$ used to approximate the strain energy: $W_{\infty} \approx W_{\mathrm{N}}$. This plot illustrates that $r_{K}$ fully stabilises to a constant value for $N \geqslant 3$.
Similar results were observed for other configurations of the SCB test. Since we have determined the first five coefficients of the crack tip asymptotic field, we choose to use $N=5$ to obtain $r_{K}: W_{\infty} \approx W_{5}$ in Eq. (11).

The procedure of determining $r_{K}$ is as follows: The strain energies $W_{1}$ and $W_{5}$ are evaluated by employing the stress field in Eq. (7) along with Eq. (10) and the normalised crack parameters in Tables 2, A.1-A.5. Then, Eq. (11) is formulated in terms of the unknown $r_{K} / a$ and is solved using a recursive algorithm. Fig. 5 presents the results of such analyses, where for all six different test configurations given in Table 1 , the calculated parameter $r_{K} / a$ is multiplied by the corresponding $a / R$ to determine $r_{K} / R$.

Evaluating the results presented in Fig. 5 shows that the data points fall in the range $0.016 \leqslant r_{K} / R \leqslant 0.043$. As is seen, $r_{K}$ is significantly dependent on geometry and material factors. However, we suggest to consider the average value of $r_{K} / R=0.03$ as a representative figure suitable for all cases. One may argue that the maximum and minimum data points in Fig. $5\left(r_{K} / R=0.043\right.$ and $\left.r_{K} / R=0.016\right)$ are significantly different from the suggested value of $r_{K} / R=0.03$. Our justification is that replacing the maximum and minimum points by the average value $r_{K} / R=0.03$ results in the change of the predefined value of 0.1 in Eq. (11) by only $\pm 5 \%$. Therefore, $r_{K} / R=0.03$ can be accepted as a very good representative value that simply works for all SCB test configurations.

We also note that multiplying the values in Fig. 5 by the respective $R / a$ gives the following range of K-dominant region as a function of crack length: $0.027 \leqslant r_{K} / a \leqslant 0.107$. Let us consider that the LEFM theory is valid when the process zone is smaller than a one-tenth of the crack length: $L_{\mathrm{FPZ}} / a<0.1$ [56]. This indicates that the size constraint applied by the K-dominant also satisfies the LEFM threshold as long as $L_{\mathrm{FPZ}}<r_{K}$. Note that $L_{\mathrm{FPZ}}<r_{K}$ is an important criterion that validates the application of a single-parameter fracture growth criterion.

\section{Discussion on the size requirement of the SCB test}

In order to have a valid measurement of the mode I fracture toughness $\left(K_{\text {Ic }}\right)$, the FPZ must lie within the K-dominant region. The reason is that the energy dissipation that forms new fracture surfaces occurs within the FPZ, and therefore for $K_{\mathrm{Ic}}$ to be reliable, $K_{\mathrm{I}}$ must be sufficient to characterise stress within the FPZ. To satisfy this condition, a minimum sample size is often recommended for fracture toughness tests, with $D \geqslant 2\left(K_{\mathrm{Ic}} / \sigma_{t}\right)^{2}$ being the minimum ISRM-suggested diameter for the SCB test and $\sigma_{t}$ being the tensile strength of the rock mass [1]. However, this size constraint is apparently based on rather limited experiment data on Colorado oil shale [9,1], and may not apply to all types of rocks. Here, we explain why this minimum size requirement 

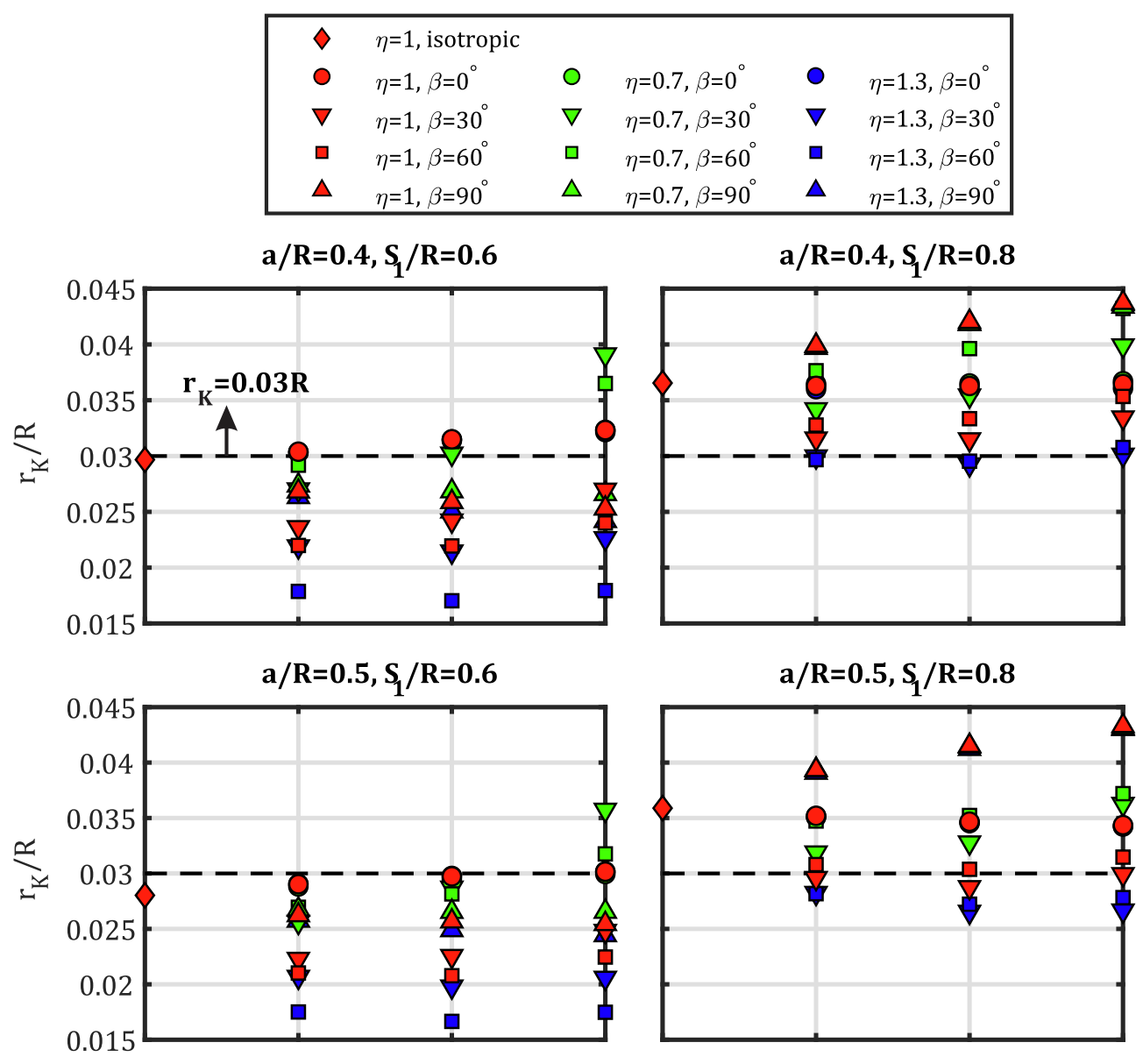

$\mathrm{a} / \mathrm{R}=0.5, \mathrm{~S}_{1} / \mathrm{R}=0.8$
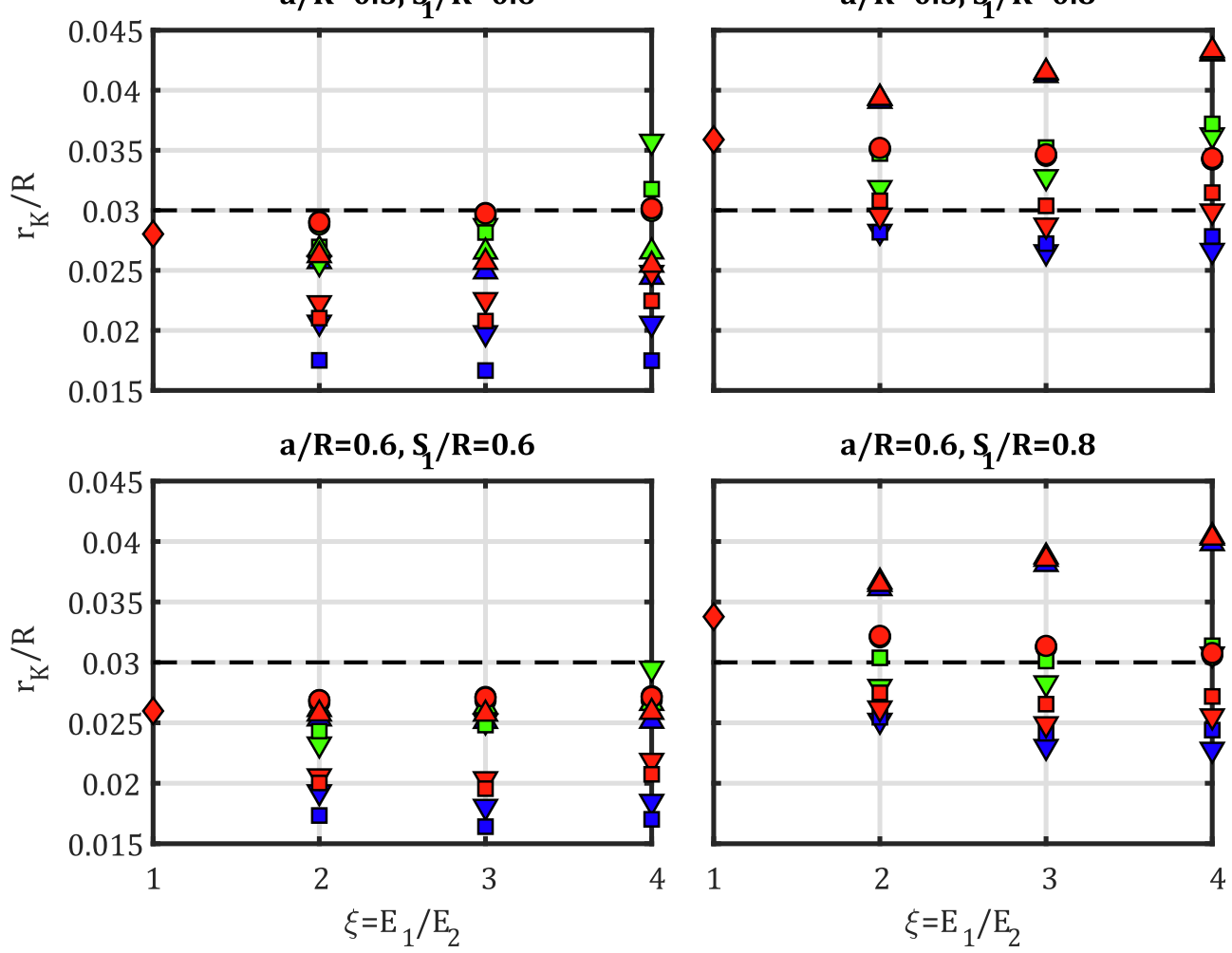

$\mathrm{a} / \mathrm{R}=0.6, \mathrm{~S}_{1} / \mathrm{R}=0.8$

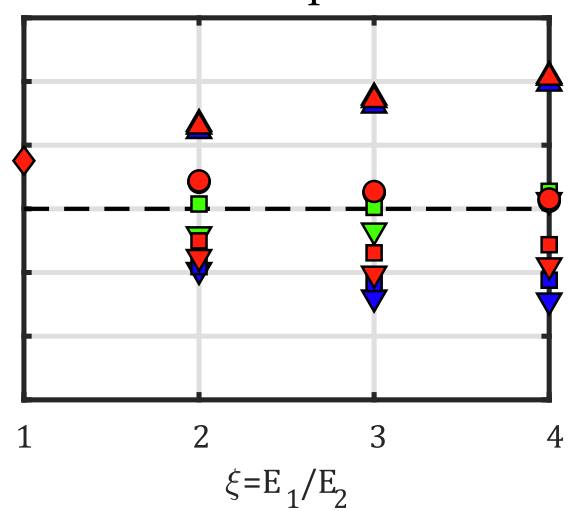

Fig. 5. The ratio of the K-dominant zone radius to the SCB disk radius, $r_{K} / R$, for various values of $a / R, S_{1} / R, \eta=G_{12} / G_{12}^{s v}, \xi=E_{1} / E_{2}$ and anisotropy angle $\beta$.

may fail to guarantee a reliable fracture toughness measurement for rocks.

Recent experiment data shows that the FPZ length in rocks, forming in a semi-elliptical shape, can be large (e.g. as large as $1 \mathrm{~cm}$ in Grimsel Granite [15]), which makes it potentially lying out of the typical Kdominant region of the SCB sample. Wei et al. [57] also approved the existence of a large process zone in fracture toughness testing of Dazhou sandstone using the cracked chevron notched Brazilian disc (CCNBD) specimen. Dutler et al. [15] compared the experimental measurements of the FPZ length with different theoretical models, and concluded that the FPZ length sits in between the values calculated by the strip-yield uniform and linear traction models. Combining these suggested bounds of the FPZ length, and the minimum size recommended by [1], one obtains the following conditions for the FPZ size and the sample size:
$\left\{\begin{array}{l}\frac{\pi}{8}\left(\frac{K_{\mathrm{Ic}}}{\sigma_{t}}\right)^{2} \leqslant L_{\mathrm{FPZ}} \leqslant \frac{9 \pi}{32}\left(\frac{K_{\mathrm{Ic}}}{\sigma_{t}}\right)^{2} \\ \left(\frac{K_{\mathrm{Ic}}}{\sigma_{t}}\right)^{2} \leqslant R\end{array}\right.$

Let us now take the minimum recommended size of the SCB sample, i.e. $R=\left(K_{\mathrm{Ic}} / \sigma_{t}\right)^{2}$. Substituting this relation into the lower and upper bounds of the FPZ length in Eq. (13) gives

$0.39 \leqslant \frac{L_{\mathrm{FPZ}}}{R} \leqslant 0.88$

Eq. (14) suggests that, for the smallest SCB sample that passes the ISRMsuggested size requirement, the FPZ length is in fact comparable to the sample radius, i.e. far greater than the size of K-dominant zone obtained in this study $\left(r_{K} / R=0.03\right)$. Here we intend to schematically compare 


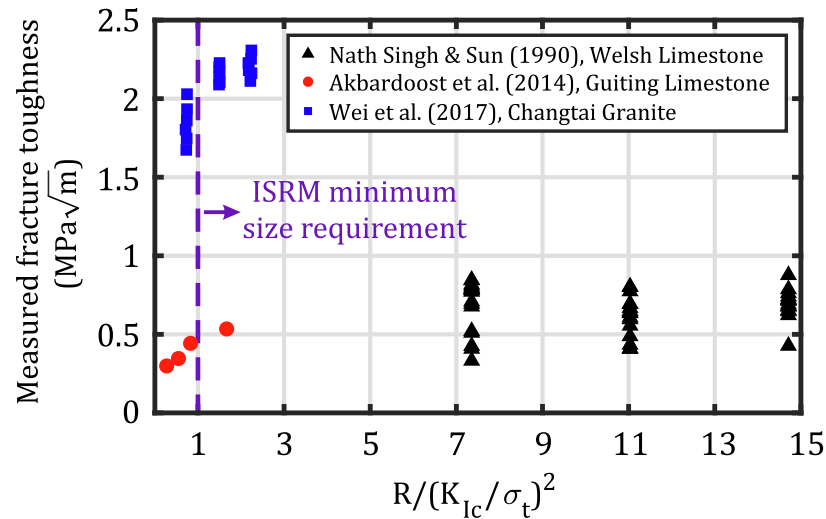

Fig. 6. Specimen size affecting the fracture toughness of rock materials: Literature data. The ISRM-suggested size requirement fails to maintain stability in fracture toughness values.

the FPZ size with the K-zone for the case $L_{\mathrm{FPZ}} / R=0.39$. Note that when the FPZ positions outside the K-dominant region, the measured $K_{\text {Ic }}$ may no longer be reliable since not only $K_{\mathrm{I}}$ but also subsequent higher order terms have contributions to the stress in the FPZ. This implies that the minimum size requirement originally put forward by Chong et al. [9] and adopted by Kuruppu et al. [1] is relatively small, and may not be an appropriate choice for all different types of rocks.

Nath Singh and Sun [58], Akbardoost et al. [4] and Wei et al. [6] have shown that an SCB test on a small sample may result in the underestimation of the fracture toughness of rocks. Fig. 6 visualises three sets of experimental data reported in these articles, plotting fracture toughness values against the normalised sample size $R /\left(K_{\mathrm{Ic}} / \sigma_{t}\right)^{2}$. The vertical line at $R /\left(K_{\mathrm{Ic}} / \sigma_{t}\right)^{2}=1$ represents the minimum suggested size put forward by the ISRM [1]. This plot demonstrates that mode I fracture toughness values do not reach a plateau at the minimum ISRMsuggested size. In fact, the tests performed on ISRM-suggested minimum size yield underestimated values of fracture toughness, as also pointed out in Ref. [59]. On the other hand, at larger sizes (in the range of $\left.11<R /\left(K_{\mathrm{Ic}} / \sigma_{t}\right)^{2}<15\right)$, the black triangular data points pertaining to Welsh limestone show practically stabilized values. We therefore conclude that the size requirement given by Kuruppu et al. [1] is not accurate, and tests performed on that basis may underestimate the true fracture toughness.

To further assess this topic, let us consider two SCB specimens, made of isotropic and anisotropic rocks, as shown in Fig. 7. Although mode I loading occurs in both cases, the isotropic specimen (with symmetrical supports, Fig. 7a) propagates in a self-planar manner, while the anisotropic specimen (with asymmetrical supports, Fig. 7b) tends to kink towards the planes with weaker strengths [16]. For example, in the case illustrated in Fig. 7b, the bedding/foliation is oriented at $\beta=60^{\circ}$ and therefore the vertical crack tends to deviate towards $\theta=60^{\circ}$. We here chose the kink angle of $\theta=30^{\circ}$, based on experimental observations in anisotropic Grimsel Granite [16]. We note that the FPZs of rocks are oriented along the direction of fracture growth, and are often of semi-elliptical shape with the width to the length ratio of $W / L=0.5$ [15]. We also assume in Fig. 7 that the size of the FPZ, when fully developed and no factor constrains its development (shown in pink colour), follows the lower bound estimate of Eq. (14) $\left(L_{\mathrm{FPZ}} / R=0.39\right)$.

Fig. 7 also presents the variations of the circumferential stress $\sigma_{\theta}$ against the radial distance $r$ that originates from the crack tip and reaches up to the boundary of FPZ. The red solid lines are directly-
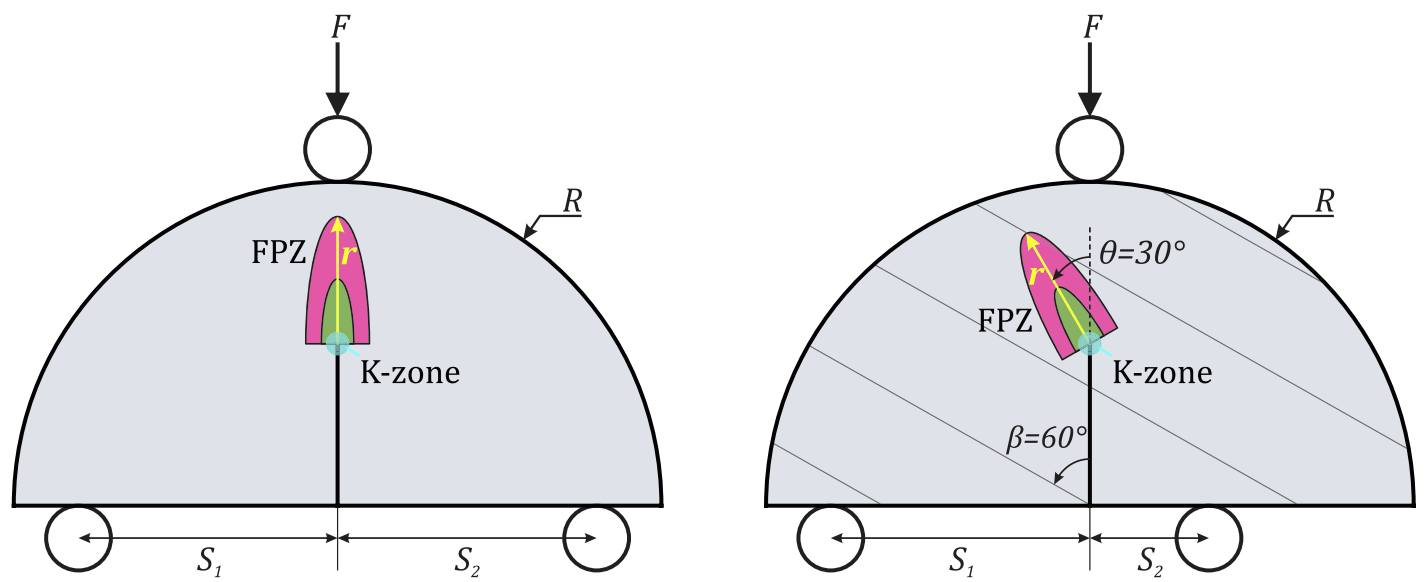

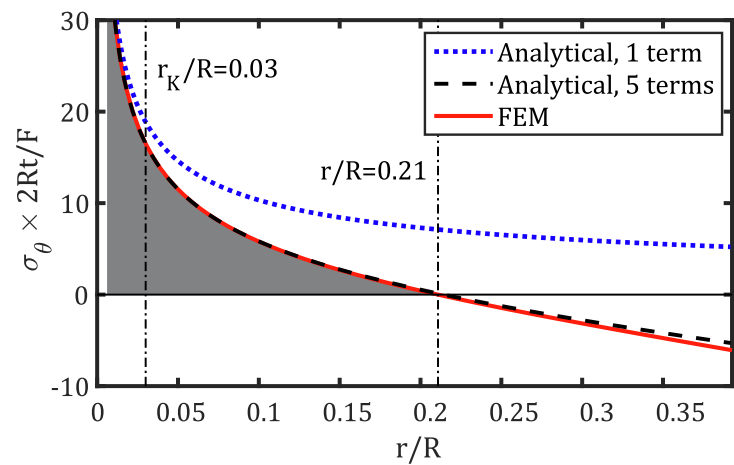

(a)

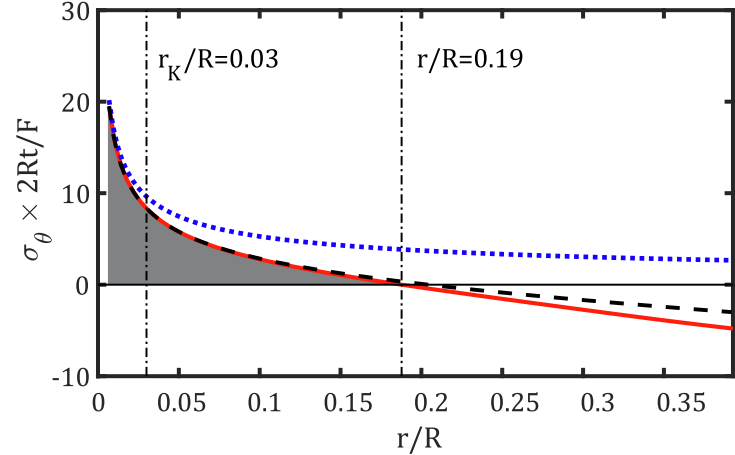

(b)

Fig. 7. Large FPZ size compared to the sample size in the smallest SCB sample that passes the ISRM-suggested size requirement $\left(R=\left(K_{\mathrm{Ic}} / \sigma_{t}\right)^{2}\right)$. The FPZ size follows the strip-yield uniform traction model. Circumferential stresses are plotted along the FPZ for the geometrical configuration of $a / R=0.5$ and $S_{1} / R=0.8$ : (a) isotropic material; (b) anisotropic material with $\eta=1.3, \xi=4$ and $\beta=60^{\circ}$. The pink area shows the FPZ expected to form in its full-development, while the green area is the region where the FPZ is constrained to develop. 


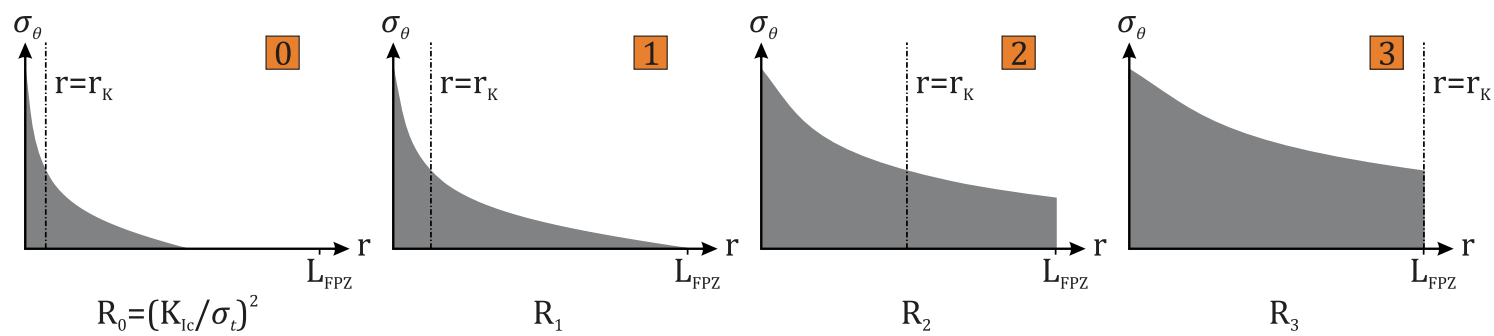

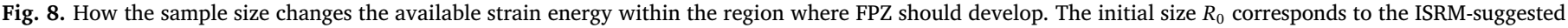
minimum size. The FPZ fully develops only when the disk radius is raised to a proper amount $R_{3}\left(R_{0}<R_{1}<R_{2}<R_{3}\right)$.

calculated FE stresses, while the blue dotted and black dashed lines correspond to the analytical solutions that include one and five stress terms, respectively. Also shown are vertical lines that represent the limit of the K-dominant zone $(r / R=0.03)$, in which the singular solution, the solution based on five terms and the FE directly-calculated results all match. Beyond the K-zone, the singular solution diverges from the FE results considerably, reaching significant discrepancies at the FPZ boundary with the error values of $185 \%$ and $155 \%$ for isotropic and anisotropic cases, respectively. These significant errors indicate the inapplicability of the singular solution and K-based propagation criteria to predict fracture growth in such small SCB samples. Note that the analytical solution with five terms, however, provides suitable estimates of stress that matches well with the FE directly-calculated results.

Another important remark about Fig. 7 is that the circumferential stress $\left(\sigma_{\theta}\right)$, which is often considered to be the driving force in fracture growth, changes its sign and becomes compressive at about $r / R=0.2$, i.e. in the middle of the region in which a fully-developed FPZ is expected to occur. This indicates that the compressive stress beyond $r / R=0.2$ does not allow the formation of micro-cracks, and thus significantly constrains the development of FPZ in full ahead of the crack tip. Consequently, the largest FPZ that can develop is constrained to the area where tensile stress is applied (coloured in green on the SCB specimens). This partially-developed FPZ embodies the region where tensile circumferential stress is present i.e. the region in grey colour in the plots. As the SCB radius $R$ increases, the distance $r$ at which $\sigma_{\theta}=0$ also grows, thereby the FPZ is allowed to approach its full-development.

Fig. 8, with plots of $\sigma_{\theta}$ versus $r$, explains how increase in the SCB specimen size allows the full development of the FPZ in a larger extent. Graph 0 shows the case where the SCB radius is the smallest possible size that meets ISRM-suggested requirement $R=R_{0}=\left(K_{\mathrm{Ic}} / \sigma_{t}\right)^{2}$. As discussed, such sample size yields an FPZ that is not developed to its full extent. Once the radius is raised to $R_{1}$, the tangential stress become tensile over the size of a full-developed FPZ. However, the K-dominant region is small compared to the size of the FPZ, and therefore the higher order parameters, that are compressive in nature, can prevent the micro-cracking process to its full extent. The larger the radius, the higher accumulation of tensile stress in the FPZ. Graph 2 shows that larger specimen accumulates more tensile stress in the FPZ, but this accumulation has not yet reached its ultimate value.

Therefore, a valid measurement of fracture toughness requires a specimen size (graph 3) where the FPZ fits inside the K-dominant zone and the accumulation of tensile stress in the FPZ reaches its peak. With the gradual increase of $R$ from graph 0 to graph 3 in Fig. 8, it is observed that the shaded area is constantly augmenting, which can be interpreted as the augmentation of strain energy. A small SCB specimen stores small strain energy in the region that FPZ can potentially develop, and therefore underestimates the fracture toughness. A valid fracture toughness can be determined from Fig. 8-3 at which further increase in $R$ does not alter the strain energy available to develop the
FPZ in its full extent. Only at such size does $K_{\text {Ic }}$ stabilise. However, cores extracted from boreholes drilled for geomechanical projects have a limited diameter up to two-three inches, thus not practically allowing sufficient size increase in the SCB samples prepared from those cores. This emphasises the need for consideration of size effect in the SCB test and to apply suitable correction factors to the fracture toughness measurements when employing small SCB samples. We note that the theoretical findings in this paper must be verified using a comprehensive experimental work where fracture toughness of different rock types (with different FPZ sizes) is measured using a large range of SCB sample sizes. This is the subject of our future research.

\section{Conclusions}

This paper reports the first five coefficients of the crack tip asymptotic field for the mode I SCB test of isotropic and anisotropic rocks, using a variety of geometrical and anisotropy parameters. Employing these calculated parameters, we then discuss the size of the K-dominant zone and compare it to the typical FPZ size of rocks. We idealise the K-dominant region as a disk centred at the crack tip with its radius being determined using an energy-based criterion. We found $r_{K}=0.03 R$ to be the average of the K-zone size in the SCB sample, a representative value that works well for a wide spectrum of anisotropy and geometrical configurations of the mode I SCB test. The comparison of the K-zone size with the typical values of the FPZ shows that the ISRM-suggested minimum sample size can largely underestimate the true $K_{\text {Ic }}$ value for rocks with large FPZ sizes. This is because the higher order terms of crack tip asymptotic field can in fact restrict the fulldevelopment of the FPZ when the FPZ is large compared to the specimen size. In order to obtain reliable fracture toughness measurements from the SCB specimen, one therefore needs to either increase the specimen size up to the limit that K-zone boundary conforms to the FPZ boundary, or to add correction factors in order to compromise on the undeveloped FPZ in small samples. This can be done for example by using size effect laws which have been proposed for obtaining the value of size-independent fracture toughness from fracture tests performed on a number of small size samples.

\section{Declaration of Competing Interest}

The authors declare that they have no known competing financial interests or personal relationships that could have appeared to influence the work reported in this paper.

\section{Acknowledgement}

The first author would like to thank the Swiss Competence Center for Energy Research - Supply of Electricity (SCCER-SoE) for its financial support. 


\section{Appendix A. Normalised crack parameters for the SCB specimen}

Tables A.1-A.5 give the normalised values of the first five coefficients of the crack tip asymptotic field for the Mode I SCB test with different geometrical and anisotropy configurations. All the values are calculated via the FEOD method, within which convergence studies were performed to guarantee the high accuracy of the reported values.

Table A.1

Normalised crack tip parameters $A_{n}^{*}, B_{n}^{*}(\mathrm{n}=1,3,4,5)$ and $T^{*}$ for the SCB specimen under mode I fracture: $a / R=0.4$ and $S_{1} / R=0.8$.

\begin{tabular}{|c|c|c|c|c|c|c|c|c|c|c|c|c|}
\hline$\eta$ & $\xi$ & $\beta\left(^{\circ}\right)$ & $S_{2} / S_{1}$ & $A_{1}^{*}$ & $B_{1}^{*}$ & $T^{*}$ & $A_{3}^{*}$ & $B_{3}^{*}$ & $A_{4}^{*}$ & $B_{4}^{*}$ & $A_{5}^{*}$ & $B_{5}^{*}$ \\
\hline \multirow[t]{13}{*}{1} & 1 & - & 1.00 & 1.9210 & 0.0000 & -0.0202 & -2.5840 & 0.0000 & -0.0840 & 0.0000 & -0.6285 & 0.0000 \\
\hline & \multirow[t]{4}{*}{2} & 0 & 1.00 & 1.9306 & 0.0000 & -0.1222 & -2.4684 & 0.0000 & 0.1839 & 0.0000 & -0.7692 & 0.0000 \\
\hline & & 30 & 0.52 & 1.2269 & 0.0000 & -0.3664 & -1.6537 & -0.5472 & -0.3786 & -0.0827 & 0.0312 & 0.3077 \\
\hline & & 60 & 0.61 & 1.3604 & 0.0000 & -0.2714 & -1.9265 & -0.4804 & -0.3804 & 0.0171 & 0.1316 & 0.3576 \\
\hline & & 90 & 1.00 & 1.9329 & 0.0000 & 0.1342 & -2.8579 & 0.0000 & -0.0071 & 0.0000 & -0.3959 & 0.0000 \\
\hline & \multirow[t]{4}{*}{3} & 0 & 1.00 & 1.9405 & 0.0000 & -0.1491 & -2.4402 & 0.0000 & 0.2463 & 0.0000 & -0.8272 & 0.0000 \\
\hline & & 30 & 0.40 & 1.0052 & 0.0000 & -0.4438 & -1.4132 & -0.7579 & -0.6315 & -0.1493 & 0.3289 & 0.6400 \\
\hline & & 60 & 0.53 & 1.2268 & 0.0000 & -0.3113 & -1.8493 & -0.6506 & -0.5665 & 0.0542 & 0.5130 & 0.6902 \\
\hline & & 90 & 1.00 & 1.9584 & 0.0000 & 0.2416 & -3.1418 & 0.0000 & -0.0540 & 0.0000 & -0.1965 & 0.0000 \\
\hline & \multirow[t]{4}{*}{4} & 0 & 1.00 & 1.9482 & 0.0000 & -0.1498 & -2.4302 & 0.0000 & 0.2922 & 0.0000 & -0.8618 & 0.0000 \\
\hline & & 30 & 0.35 & 0.9020 & 0.0001 & -0.5064 & -1.3076 & -0.8770 & -0.8045 & -0.1731 & 0.5072 & 0.9523 \\
\hline & & 60 & 0.49 & 1.1740 & 0.0000 & -0.3394 & -1.8531 & -0.7425 & -0.6509 & 0.1365 & 0.7465 & 0.9536 \\
\hline & & 90 & 1.00 & 1.9883 & 0.0000 & 0.3227 & -3.4225 & 0.0000 & -0.0833 & 0.0000 & -0.0189 & 0.0000 \\
\hline \multirow[t]{12}{*}{0.7} & \multirow[t]{4}{*}{2} & 0 & 1.00 & 1.9415 & 0.0000 & -0.0909 & -2.5272 & 0.0000 & 0.1338 & 0.0000 & -0.7372 & 0.0000 \\
\hline & & 30 & 0.49 & 1.1588 & 0.0001 & -0.3668 & -1.5576 & -0.5483 & -0.4376 & -0.1589 & 0.0640 & 0.2958 \\
\hline & & 60 & 0.67 & 1.4623 & -0.0001 & -0.2512 & -2.1065 & -0.5209 & -0.3357 & 0.0030 & 0.1448 & 0.3967 \\
\hline & & 90 & 1.00 & 1.9625 & 0.0000 & 0.1817 & -2.9959 & 0.0000 & -0.0264 & 0.0000 & -0.3640 & 0.0000 \\
\hline & \multirow[t]{4}{*}{3} & 0 & 1.00 & 1.9490 & 0.0000 & -0.1188 & -2.4902 & 0.0000 & 0.1864 & 0.0000 & -0.7962 & 0.0000 \\
\hline & & 30 & 0.39 & 0.9724 & -0.0001 & -0.4353 & -1.3737 & -0.7811 & -0.7417 & -0.2625 & 0.4031 & 0.6827 \\
\hline & & 60 & 0.57 & 1.3162 & 0.0000 & -0.3051 & -2.0222 & -0.7109 & -0.5416 & 0.0169 & 0.6103 & 0.7730 \\
\hline & & 90 & 1.00 & 1.9986 & 0.0000 & 0.2987 & -3.3312 & 0.0000 & -0.0619 & 0.0000 & -0.1710 & 0.0000 \\
\hline & \multirow[t]{4}{*}{4} & 0 & 1.00 & 1.9552 & 0.0000 & -0.1204 & -2.4754 & 0.0000 & 0.2244 & 0.0000 & -0.8309 & 0.0000 \\
\hline & & 30 & 0.35 & 0.8860 & 0.0000 & -0.4837 & -1.3039 & -0.9312 & -0.9808 & -0.3330 & 0.6552 & 1.0773 \\
\hline & & 60 & 0.54 & 1.2544 & 0.0000 & -0.3417 & -2.0171 & -0.8112 & -0.6253 & 0.0992 & 0.8769 & 1.0564 \\
\hline & & 90 & 1.00 & 2.0375 & 0.0000 & 0.3873 & -3.6592 & 0.0000 & -0.0824 & 0.0000 & 0.0020 & 0.0000 \\
\hline \multirow[t]{12}{*}{1.3} & \multirow[t]{4}{*}{2} & 0 & 1.00 & 1.9265 & 0.0000 & -0.1386 & -2.4458 & 0.0000 & -0.2186 & 0.0000 & -0.7841 & 0.0000 \\
\hline & & 30 & 0.54 & 1.2783 & 0.0000 & -0.3660 & -1.7411 & -0.5535 & 0.3450 & 0.0343 & 0.0184 & 0.3197 \\
\hline & & 60 & 0.57 & 1.2952 & 0.0000 & -0.2814 & -1.8304 & -0.4610 & 0.4082 & -0.0174 & 0.1333 & 0.3330 \\
\hline & & 90 & 1.00 & 1.9188 & 0.0000 & 0.1100 & -2.7971 & 0.0000 & -0.0084 & 0.0000 & -0.4148 & 0.0000 \\
\hline & \multirow[t]{4}{*}{3} & 0 & 1.00 & 1.9378 & 0.0000 & -0.1661 & -2.4225 & 0.0000 & -0.2872 & 0.0000 & -0.8401 & 0.0000 \\
\hline & & 30 & 0.41 & 1.0310 & 0.0000 & -0.4511 & -1.4608 & -0.7543 & 0.5703 & 0.0813 & 0.2911 & 0.6164 \\
\hline & & 60 & 0.50 & 1.1729 & 0.0000 & -0.3144 & -1.7671 & -0.6226 & 0.5883 & -0.0632 & 0.4760 & 0.6501 \\
\hline & & 90 & 1.00 & 1.9382 & 0.0000 & 0.2119 & -3.0557 & 0.0000 & 0.0472 & 0.0000 & -0.2082 & 0.0000 \\
\hline & \multirow[t]{4}{*}{4} & 0 & 1.00 & 1.9463 & 0.0000 & -0.1674 & -2.4153 & 0.0000 & -0.3386 & 0.0000 & -0.8739 & -0.0001 \\
\hline & & 30 & 0.35 & 0.9152 & 0.0001 & -0.5227 & -1.3317 & -0.8572 & 0.7058 & 0.0803 & 0.4246 & 0.8742 \\
\hline & & 60 & 0.47 & 1.1266 & 0.0001 & -0.3383 & -1.7807 & -0.7119 & 0.6783 & -0.1438 & 0.6996 & 0.9113 \\
\hline & & 90 & 1.00 & 1.9628 & 0.0000 & 0.2885 & -3.3122 & 0.0000 & 0.0836 & 0.0000 & -0.0224 & 0.0000 \\
\hline
\end{tabular}

Table A.2

Normalised crack tip parameters $A_{n}^{*}, B_{n}^{*}(\mathrm{n}=1,3,4,5)$ and $T^{*}$ for the SCB specimen under mode I fracture: $a / R=0.5$ and $S_{1} / R=0.6$.

\begin{tabular}{|c|c|c|c|c|c|c|c|c|c|c|c|c|}
\hline$\eta$ & $\xi$ & $\beta\left({ }^{\circ}\right)$ & $S_{2} / S_{1}$ & $A_{1}^{*}$ & $B_{1}^{*}$ & $T^{*}$ & $A_{3}^{*}$ & $B_{3}^{*}$ & $A_{4}^{*}$ & $B_{4}^{*}$ & $A_{5}^{*}$ & $B_{5}^{*}$ \\
\hline \multirow[t]{13}{*}{1} & 1 & - & 1.00 & 1.6205 & 0.0000 & -0.4645 & -3.0625 & 0.0000 & 0.3083 & 0.0000 & -0.8427 & 0.0000 \\
\hline & \multirow[t]{4}{*}{2} & 0 & 1.00 & 1.6522 & 0.0000 & -0.3277 & -3.0893 & 0.0000 & -0.1348 & 0.0000 & -1.0582 & 0.0000 \\
\hline & & 30 & 0.60 & 1.1514 & 0.0000 & -1.1323 & -2.0455 & -0.4780 & -0.5787 & 0.2068 & -0.4003 & 0.4004 \\
\hline & & 60 & 0.68 & 1.2274 & 0.0001 & -1.0697 & -2.2005 & -0.3863 & -0.5819 & 0.3474 & -0.3732 & 0.4020 \\
\hline & & 90 & 1.00 & 1.5928 & 0.0000 & -0.5338 & -3.0934 & 0.0000 & -0.4340 & 0.0000 & -0.6132 & 0.0000 \\
\hline & \multirow[t]{4}{*}{3} & 0 & 1.00 & 1.6692 & 0.0000 & -0.2161 & -3.1093 & 0.0000 & -0.0287 & 0.0000 & -1.1630 & 0.0000 \\
\hline & & 30 & 0.48 & 0.9823 & 0.0000 & -1.4102 & -1.7312 & -0.6156 & -0.6088 & 0.3071 & -0.2864 & 0.6085 \\
\hline & & 60 & 0.61 & 1.1262 & -0.0001 & -1.2236 & -2.0271 & -0.4720 & -0.5409 & 0.6243 & -0.2994 & 0.5610 \\
\hline & & 90 & 1.00 & 1.5852 & 0.0000 & -0.5326 & -3.1934 & 0.0000 & -0.4722 & 0.0000 & -0.4740 & 0.0000 \\
\hline & \multirow[t]{4}{*}{4} & 0 & 1.00 & 1.6802 & 0.0000 & -0.1154 & -3.1237 & 0.0000 & 0.0449 & 0.0000 & -1.2255 & 0.0000 \\
\hline & & 30 & 0.42 & 0.8955 & -0.0001 & -1.5923 & -1.5765 & -0.6672 & -0.5706 & 0.3984 & -0.2606 & 0.7374 \\
\hline & & 60 & 0.57 & 1.0820 & 0.0000 & -1.2909 & -1.9836 & -0.5086 & -0.4421 & 0.8724 & -0.2988 & 0.6287 \\
\hline & & 90 & 1.00 & 1.5869 & 0.0000 & -0.5114 & -3.3312 & 0.0000 & -0.4838 & 0.0000 & -0.3600 & 0.0000 \\
\hline \multirow[t]{4}{*}{0.7} & \multirow[t]{4}{*}{2} & 0 & 1.00 & 1.6522 & 0.0000 & -0.4349 & -3.0769 & 0.0000 & -0.1955 & 0.0000 & -1.0470 & 0.0000 \\
\hline & & 30 & 0.57 & 1.0975 & 0.0000 & -1.1778 & -1.9195 & -0.4639 & -0.6619 & 0.1127 & -0.2885 & 0.4556 \\
\hline & & 60 & 0.75 & 1.3251 & 0.0001 & -0.9379 & -2.4561 & -0.4133 & -0.5066 & 0.4468 & -0.4019 & 0.4627 \\
\hline & & 90 & 1.00 & 1.6129 & 0.0000 & -0.5219 & -3.2059 & 0.0000 & -0.4245 & 0.0000 & -0.6552 & 0.0000 \\
\hline
\end{tabular}

(continued on next page) 
Table A.2 (continued)

\begin{tabular}{|c|c|c|c|c|c|c|c|c|c|c|c|c|}
\hline$\eta$ & $\xi$ & $\beta\left({ }^{\circ}\right)$ & $S_{2} / S_{1}$ & $A_{1}^{*}$ & $B_{1}^{*}$ & $T^{*}$ & $A_{3}^{*}$ & $B_{3}^{*}$ & $A_{4}^{*}$ & $B_{4}^{*}$ & $A_{5}^{*}$ & $B_{5}^{*}$ \\
\hline & 3 & 0 & 1.00 & 1.6669 & 0.0000 & -0.3571 & -3.0886 & 0.0000 & -0.1129 & 0.0000 & -1.1348 & 0.0000 \\
\hline & & 30 & 0.47 & 0.9565 & -0.0001 & -1.4339 & -1.6473 & -0.5922 & -0.7085 & 0.2162 & -0.1661 & 0.7540 \\
\hline & & 60 & 0.66 & 1.2105 & 0.0000 & -1.1213 & -2.2584 & -0.5207 & -0.4601 & 0.7480 & -0.3192 & 0.6033 \\
\hline & & 90 & 1.00 & 1.6169 & 0.0000 & -0.4879 & -3.3920 & 0.0000 & -0.4531 & 0.0000 & -0.5051 & -0.0001 \\
\hline & 4 & 0 & 1.00 & 1.6768 & 0.0000 & -0.2808 & -3.0999 & 0.0000 & -0.0554 & 0.0000 & -1.1877 & 0.0000 \\
\hline & & 30 & 0.43 & 0.8848 & 0.0000 & -1.5996 & -1.5123 & -0.6419 & -0.6823 & 0.3116 & -0.1257 & 0.9552 \\
\hline & & 60 & 0.62 & 1.1575 & 0.0000 & -1.2041 & -2.2053 & -0.5739 & -0.3534 & 1.0089 & -0.3141 & 0.6542 \\
\hline & & 90 & 1.00 & 1.6285 & 0.0000 & -0.4462 & -3.6072 & 0.0000 & -0.4619 & 0.0000 & -0.3699 & 0.0000 \\
\hline \multirow[t]{12}{*}{1.3} & 2 & 0 & 1.00 & 1.6548 & 0.0000 & -0.2579 & -3.1181 & 0.0000 & 0.0897 & 0.0000 & -1.0624 & 0.0000 \\
\hline & & 30 & 0.62 & 1.1924 & 0.0000 & -1.1052 & -2.1520 & -0.4913 & 0.5302 & -0.2695 & -0.4588 & 0.3831 \\
\hline & & 60 & 0.64 & 1.1684 & 0.0000 & -1.1456 & -2.0657 & -0.3736 & 0.6242 & -0.2810 & -0.3425 & 0.3803 \\
\hline & & 90 & 1.00 & 1.5830 & 0.0000 & -0.5396 & -3.0524 & 0.0000 & 0.4452 & 0.0000 & -0.5579 & 0.0000 \\
\hline & 3 & 0 & 1.00 & 1.6731 & 0.0000 & -0.1269 & -3.1413 & 0.0000 & -0.0351 & 0.0000 & -1.1812 & 0.0000 \\
\hline & & 30 & 0.49 & 1.0030 & 0.0001 & -1.4006 & -1.8059 & -0.6380 & 0.5544 & -0.3649 & -0.3437 & 0.5429 \\
\hline & & 60 & 0.57 & 1.0775 & 0.0001 & -1.2818 & -1.9129 & -0.4472 & 0.5888 & -0.5466 & -0.2729 & 0.5588 \\
\hline & & 90 & 1.00 & 1.5679 & 0.0000 & -0.5609 & -3.0992 & 0.0000 & 0.4908 & 0.0000 & -0.4184 & 0.0000 \\
\hline & 4 & 0 & 1.00 & 1.6848 & 0.0000 & -0.0127 & -3.1566 & 0.0000 & -0.1215 & 0.0000 & -1.2511 & 0.0000 \\
\hline & & 30 & 0.42 & 0.9053 & 0.0000 & -1.5929 & -1.6373 & -0.6938 & 0.5130 & -0.4498 & -0.3194 & 0.6357 \\
\hline & & 60 & 0.55 & 1.0390 & 0.0000 & -1.3410 & -1.8776 & -0.4741 & 0.4958 & -0.7906 & -0.2731 & 0.6423 \\
\hline & & 90 & 1.00 & 1.5631 & 0.0000 & -0.5541 & -3.1881 & 0.0000 & 0.5040 & 0.0000 & -0.3149 & 0.0000 \\
\hline
\end{tabular}

Table A.3

Normalised crack tip parameters $A_{n}^{*}, B_{n}^{*}(\mathrm{n}=1,3,4,5)$ and $T^{*}$ for the SCB specimen under mode I fracture: $a / R=0.5$ and $S_{1} / R=0.8$.

\begin{tabular}{|c|c|c|c|c|c|c|c|c|c|c|c|c|}
\hline$\eta$ & $\xi$ & $\beta\left({ }^{\circ}\right)$ & $S_{2} / S_{1}$ & $A_{1}^{*}$ & $B_{1}^{*}$ & $T^{*}$ & $A_{3}^{*}$ & $B_{3}^{*}$ & $A_{4}^{*}$ & $B_{4}^{*}$ & $A_{5}^{*}$ & $B_{5}^{*}$ \\
\hline \multirow[t]{13}{*}{1} & 1 & - & 1.00 & 2.3101 & 0.0000 & 0.6492 & -4.7355 & 0.0000 & -0.2202 & 0.0000 & -1.5296 & 0.0000 \\
\hline & \multirow[t]{4}{*}{2} & 0 & 1.00 & 2.3219 & 0.0000 & 0.8719 & -4.6073 & 0.0000 & 0.4266 & 0.0000 & -1.7859 & 0.0000 \\
\hline & & 30 & 0.52 & 1.4964 & 0.0000 & -0.3171 & -2.9187 & -0.6593 & -0.3358 & 0.3106 & -0.6320 & 0.4595 \\
\hline & & 60 & 0.60 & 1.6471 & 0.0000 & -0.2354 & -3.3412 & -0.5644 & -0.3376 & 0.5186 & -0.5675 & 0.5019 \\
\hline & & 90 & 1.00 & 2.3165 & 0.0000 & 0.6145 & -5.0639 & 0.0000 & 0.0278 & 0.0000 & -1.1080 & 0.0000 \\
\hline & \multirow[t]{4}{*}{3} & 0 & 1.00 & 2.3315 & 0.0000 & 1.1165 & -4.5770 & 0.0000 & 0.5478 & 0.0000 & -1.8852 & 0.0000 \\
\hline & & 30 & 0.40 & 1.2239 & 0.0000 & -0.6494 & -2.3903 & -0.8524 & -0.4784 & 0.3926 & -0.3492 & 0.7412 \\
\hline & & 60 & 0.52 & 1.4803 & 0.0001 & -0.4500 & -3.0814 & -0.7174 & -0.3899 & 0.8510 & -0.2903 & 0.7695 \\
\hline & & 90 & 1.00 & 2.3379 & 0.0000 & 0.6605 & -5.4268 & 0.0000 & -0.0697 & 0.0000 & -0.7510 & 0.0000 \\
\hline & \multirow[t]{4}{*}{4} & 0 & 1.00 & 2.3383 & 0.0000 & 1.3542 & -4.5656 & 0.0000 & 0.6328 & 0.0000 & -1.9398 & 0.0000 \\
\hline & & 30 & 0.35 & 1.0925 & -0.0001 & -0.8671 & -2.1431 & -0.9276 & -0.4933 & 0.4790 & -0.2529 & 0.9317 \\
\hline & & 60 & 0.48 & 1.4083 & 0.0001 & -0.5599 & -3.0165 & -0.7888 & -0.3207 & 1.1577 & -0.1943 & 0.9109 \\
\hline & & 90 & 1.00 & 2.3651 & 0.0000 & 0.7154 & -5.7987 & 0.0000 & -0.1294 & 0.0000 & -0.4313 & 0.0001 \\
\hline \multirow[t]{12}{*}{0.7} & \multirow[t]{4}{*}{2} & 0 & 1.00 & 2.3260 & 0.0000 & 0.9117 & -4.6508 & 0.0000 & 0.3125 & 0.0000 & -1.7111 & 0.0000 \\
\hline & & 30 & 0.49 & 1.4229 & 0.0000 & -0.3680 & -2.7531 & -0.6470 & -0.4160 & 0.1678 & -0.5350 & 0.4834 \\
\hline & & 60 & 0.66 & 1.7675 & 0.0000 & -0.1514 & -3.6456 & -0.6185 & -0.2254 & 0.5964 & -0.6366 & 0.5829 \\
\hline & & 90 & 1.00 & 2.3424 & 0.0000 & 0.7036 & -5.2513 & 0.0000 & -0.0187 & 0.0000 & -1.0355 & 0.0000 \\
\hline & \multirow[t]{4}{*}{3} & 0 & 1.00 & 2.3326 & 0.0000 & 1.1469 & -4.6049 & 0.0000 & 0.4118 & 0.0000 & -1.8089 & 0.0000 \\
\hline & & 30 & 0.39 & 1.1932 & 0.0001 & -0.6744 & -2.3035 & -0.8421 & -0.5890 & 0.2495 & -0.2328 & 0.8802 \\
\hline & & 60 & 0.57 & 1.5848 & 0.0000 & -0.3988 & -3.3539 & -0.7922 & -0.2725 & 0.9587 & -0.3424 & 0.8524 \\
\hline & & 90 & 1.00 & 2.3762 & 0.0000 & 0.7692 & -5.7066 & 0.0000 & -0.0962 & 0.0000 & -0.6738 & -0.0001 \\
\hline & \multirow[t]{4}{*}{4} & 0 & 1.00 & 2.3377 & 0.0000 & 1.3774 & -4.5856 & 0.0000 & 0.4807 & 0.0000 & -1.8630 & 0.0000 \\
\hline & & 30 & 0.35 & 1.0830 & 0.0000 & -0.8750 & -2.0924 & -0.9247 & -0.6311 & 0.3373 & -0.1028 & 1.1775 \\
\hline & & 60 & 0.52 & 1.5013 & 0.0000 & -0.5232 & -3.2722 & -0.8784 & -0.1881 & 1.2886 & -0.2495 & 0.9753 \\
\hline & & 90 & 1.00 & 2.4140 & 0.0000 & 0.8379 & -6.1632 & 0.0000 & -0.1419 & 0.0000 & -0.3457 & 0.0000 \\
\hline \multirow[t]{12}{*}{1.3} & \multirow[t]{4}{*}{2} & 0 & 1.00 & 2.3231 & 0.0000 & 0.8540 & -4.6069 & 0.0000 & -0.5120 & 0.0000 & -1.8328 & 0.0000 \\
\hline & & 30 & 0.54 & 1.5515 & -0.0001 & -0.2814 & -3.0604 & -0.6742 & 0.2861 & -0.4074 & -0.6825 & 0.4605 \\
\hline & & 60 & 0.57 & 1.5712 & 0.0000 & -0.2831 & -3.1755 & -0.5375 & 0.4023 & -0.4651 & -0.5045 & 0.4613 \\
\hline & & 90 & 1.00 & 2.3053 & 0.0000 & 0.5681 & -4.9903 & 0.0000 & -0.0645 & 0.0000 & -1.1448 & 0.0000 \\
\hline & \multirow[t]{4}{*}{3} & 0 & 1.00 & 2.3344 & 0.0000 & 1.1022 & -4.5852 & 0.0000 & -0.6495 & 0.0000 & -1.9334 & 0.0000 \\
\hline & & 30 & 0.40 & 1.2491 & 0.0000 & -0.6346 & -2.4755 & -0.8699 & 0.4161 & -0.4848 & -0.4032 & 0.6803 \\
\hline & & 60 & 0.49 & 1.4172 & 0.0001 & -0.4802 & -2.9441 & -0.6786 & 0.4602 & -0.7837 & -0.2342 & 0.7396 \\
\hline & & 90 & 1.00 & 2.3195 & 0.0000 & 0.6012 & -5.3041 & 0.0000 & 0.0477 & 0.0000 & -0.7833 & -0.0001 \\
\hline & \multirow[t]{4}{*}{4} & 0 & 1.00 & 2.3424 & 0.0000 & 1.3425 & -4.5788 & 0.0000 & -0.7472 & 0.0000 & -1.9891 & -0.0001 \\
\hline & & 30 & 0.34 & 1.1022 & 0.0001 & -0.8631 & -2.2024 & -0.9439 & 0.4206 & -0.5643 & -0.3173 & 0.8157 \\
\hline & & 60 & 0.46 & 1.3533 & -0.0001 & -0.5831 & -2.8940 & -0.7420 & 0.4017 & -1.0810 & -0.1331 & 0.8996 \\
\hline & & 90 & 1.00 & 2.3404 & 0.0000 & 0.6469 & -5.6297 & 0.0000 & 0.1179 & 0.0000 & -0.4618 & 0.0000 \\
\hline
\end{tabular}


Table A.4

Normalised crack tip parameters $A_{n}^{*}, B_{n}^{*}(\mathrm{n}=1,3,4,5)$ and $T^{*}$ for the SCB specimen under mode I fracture: $a / R=0.6$ and $S_{1} / R=0.6$.

\begin{tabular}{|c|c|c|c|c|c|c|c|c|c|c|c|c|}
\hline$\eta$ & $\xi$ & $\beta\left({ }^{\circ}\right)$ & $S_{2} / S_{1}$ & $A_{1}^{*}$ & $B_{1}^{*}$ & $T^{*}$ & $A_{3}^{*}$ & $B_{3}^{*}$ & $A_{4}^{*}$ & $B_{4}^{*}$ & $A_{5}^{*}$ & $B_{5}^{*}$ \\
\hline \multirow[t]{13}{*}{1} & 1 & - & 1.00 & 2.1443 & 0.0000 & 0.0258 & -6.1946 & 0.0000 & -0.0595 & 0.0000 & -3.1859 & 0.0000 \\
\hline & \multirow[t]{4}{*}{2} & 0 & 1.00 & 2.1749 & 0.0000 & 0.5169 & -6.2446 & 0.0000 & 0.2348 & 0.0000 & -3.5020 & 0.0000 \\
\hline & & 30 & 0.57 & 1.4796 & 0.0000 & -1.2139 & -4.0240 & -0.6819 & -0.3857 & 0.7467 & -2.1109 & 0.3399 \\
\hline & & 60 & 0.64 & 1.5832 & 0.0000 & -1.1545 & -4.3622 & -0.5624 & -0.4137 & 0.9945 & -2.1460 & 0.3561 \\
\hline & & 90 & 1.00 & 2.1180 & 0.0000 & -0.2272 & -6.2513 & 0.0000 & -0.2944 & 0.0000 & -2.7677 & 0.0000 \\
\hline & \multirow[t]{4}{*}{3} & 0 & 1.00 & 2.1910 & 0.0000 & 0.9325 & -6.2794 & 0.0000 & 0.4186 & 0.0000 & -3.6503 & 0.0001 \\
\hline & & 30 & 0.44 & 1.2365 & 0.0001 & -1.6905 & -3.3406 & -0.8600 & -0.2786 & 0.9876 & -1.7947 & 0.3471 \\
\hline & & 60 & 0.55 & 1.4281 & 0.0000 & -1.4222 & -3.9765 & -0.6969 & -0.2668 & 1.5516 & -1.9062 & 0.4144 \\
\hline & & 90 & 1.00 & 2.1118 & 0.0000 & -0.2732 & -6.4214 & 0.0000 & -0.3978 & 0.0000 & -2.4243 & 0.0000 \\
\hline & \multirow[t]{4}{*}{4} & 0 & 1.00 & 2.2011 & 0.0000 & 1.3007 & -6.3014 & 0.0000 & 0.5482 & 0.0000 & -3.7377 & 0.0000 \\
\hline & & 30 & 0.38 & 1.1078 & 0.0000 & -1.9653 & -3.0049 & -0.9244 & -0.0942 & 1.1472 & -1.6750 & 0.2792 \\
\hline & & 60 & 0.51 & 1.3540 & -0.0001 & -1.5268 & -3.8630 & -0.7699 & -0.0710 & 1.9883 & -1.7948 & 0.4305 \\
\hline & & 90 & 1.00 & 2.1148 & 0.0000 & -0.2664 & -6.6477 & 0.0000 & -0.4570 & 0.0000 & -2.1039 & 0.0001 \\
\hline \multirow[t]{12}{*}{0.7} & \multirow[t]{4}{*}{2} & 0 & 1.00 & 2.1708 & 0.0000 & 0.4078 & -6.2013 & 0.0000 & 0.0716 & 0.0000 & -3.4513 & 0.0000 \\
\hline & & 30 & 0.54 & 1.4193 & 0.0000 & -1.3067 & -3.7963 & -0.6317 & -0.4089 & 0.5782 & -1.9944 & 0.3509 \\
\hline & & 60 & 0.70 & 1.6989 & 0.0000 & -0.9659 & -4.8053 & -0.6162 & -0.2331 & 1.2451 & -2.3692 & 0.4267 \\
\hline & & 90 & 1.00 & 2.1354 & 0.0000 & -0.1796 & -6.4198 & 0.0000 & -0.3771 & 0.0000 & -2.6911 & 0.0000 \\
\hline & \multirow[t]{4}{*}{3} & 0 & 1.00 & 2.1841 & 0.0000 & 0.7728 & -6.2149 & 0.0000 & 0.2188 & 0.0000 & -3.5846 & 0.0000 \\
\hline & & 30 & 0.44 & 1.2097 & 0.0000 & -1.7371 & -3.1873 & -0.7781 & -0.2544 & 0.8711 & -1.7644 & 0.3678 \\
\hline & & 60 & 0.60 & 1.5261 & 0.0000 & -1.2804 & -4.3779 & -0.7931 & -0.0893 & 1.8324 & -2.0920 & 0.4804 \\
\hline & & 90 & 1.00 & 2.1413 & 0.0000 & -0.1880 & -6.7222 & 0.0000 & -0.4683 & 0.0000 & -2.3010 & -0.0002 \\
\hline & \multirow[t]{4}{*}{4} & 0 & 1.00 & 2.1926 & 0.0000 & 1.1031 & -6.2272 & 0.0000 & 0.3207 & 0.0000 & -3.6605 & 0.0001 \\
\hline & & 30 & 0.39 & 1.0984 & -0.0001 & -1.9816 & -2.8852 & -0.8269 & -0.0272 & 1.0718 & -1.6978 & 0.2829 \\
\hline & & 60 & 0.55 & 1.4415 & 0.0000 & -1.4087 & -4.2503 & -0.8978 & 0.1071 & 2.2862 & -1.9406 & 0.5086 \\
\hline & & 90 & 1.00 & 2.1549 & 0.0000 & -0.1584 & -7.0687 & 0.0000 & -0.5190 & -0.0001 & -1.9391 & -0.0001 \\
\hline \multirow[t]{12}{*}{1.3} & \multirow[t]{4}{*}{2} & 0 & 1.00 & 2.1806 & 0.0000 & 0.5885 & -6.3079 & 0.0000 & -0.3554 & 0.0000 & -3.5281 & 0.0000 \\
\hline & & 30 & 0.59 & 1.5255 & -0.0001 & -1.1534 & -4.2085 & -0.7162 & 0.3567 & -0.8694 & -2.1906 & 0.3487 \\
\hline & & 60 & 0.60 & 1.5135 & 0.0000 & -1.2638 & -4.1228 & -0.5311 & 0.5079 & -0.8482 & -1.9968 & 0.3280 \\
\hline & & 90 & 1.00 & 2.1105 & 0.0000 & -0.2562 & -6.1892 & 0.0000 & 0.2326 & 0.0000 & -2.7934 & 0.0000 \\
\hline & \multirow[t]{4}{*}{3} & 0 & 1.00 & 2.1986 & 0.0000 & 1.0345 & -6.3558 & 0.0000 & -0.5698 & 0.0000 & -3.6930 & -0.0001 \\
\hline & & 30 & 0.45 & 1.2582 & 0.0000 & -1.6671 & -3.4674 & -0.9161 & 0.2782 & -1.0781 & -1.8179 & 0.3447 \\
\hline & & 60 & 0.53 & 1.3706 & 0.0000 & -1.5050 & -3.7678 & -0.6393 & 0.3574 & -1.3962 & -1.7814 & 0.3936 \\
\hline & & 90 & 1.00 & 2.0968 & 0.0000 & -0.3271 & -6.2804 & 0.0000 & 0.3412 & 0.0000 & -2.4818 & 0.0000 \\
\hline & \multirow[t]{4}{*}{4} & 0 & 1.00 & 2.2098 & 0.0000 & 1.4246 & -6.3840 & 0.0000 & -0.7229 & 0.0000 & -3.7919 & 0.0000 \\
\hline & & 30 & 0.38 & 1.1169 & 0.0000 & -1.9644 & -3.1055 & -0.9919 & 0.1202 & -1.2115 & -1.6629 & 0.2839 \\
\hline & & 60 & 0.49 & 1.3037 & 0.0000 & -1.5955 & -3.6672 & -0.6929 & 0.1592 & -1.8310 & -1.6925 & 0.4048 \\
\hline & & 90 & 1.00 & 2.0932 & 0.0000 & -0.3352 & -6.4347 & 0.0000 & 0.4034 & 0.0000 & -2.1915 & 0.0000 \\
\hline
\end{tabular}

Table A.5

Normalised crack tip parameters $A_{n}^{*}, B_{n}^{*}(\mathrm{n}=1,3,4,5)$ and $T^{*}$ for the SCB specimen under mode I fracture: $a / R=0.6$ and $S_{1} / R=0.8$.

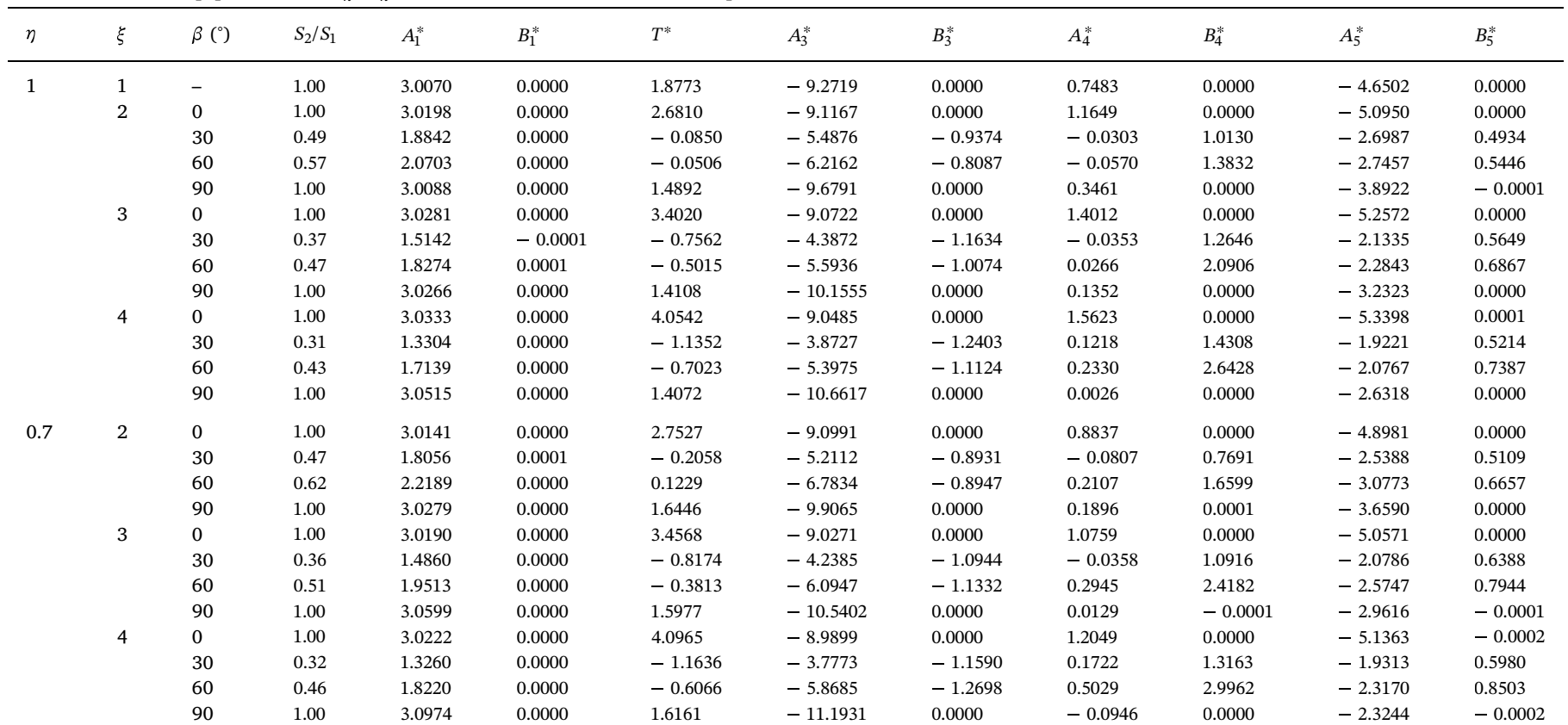

(continued on next page) 
Table A.5 (continued)

\begin{tabular}{|c|c|c|c|c|c|c|c|c|c|c|c|c|}
\hline$\eta$ & $\xi$ & $\beta\left({ }^{\circ}\right)$ & $S_{2} / S_{1}$ & $A_{1}^{*}$ & $B_{1}^{*}$ & $T^{*}$ & $A_{3}^{*}$ & $B_{3}^{*}$ & $A_{4}^{*}$ & $B_{4}^{*}$ & $A_{5}^{*}$ & $B_{5}^{*}$ \\
\hline \multirow[t]{12}{*}{1.3} & 2 & 0 & 1.00 & 3.0278 & 0.0000 & 2.6483 & -9.1778 & 0.0000 & -1.3765 & 0.0000 & -5.2203 & 0.0001 \\
\hline & & 30 & 0.51 & 1.9442 & 0.0000 & 0.0019 & -5.7205 & -0.9721 & -0.0169 & -1.1897 & -2.8006 & 0.5061 \\
\hline & & 60 & 0.53 & 1.9780 & 0.0000 & -0.1535 & -5.9015 & -0.7617 & 0.2023 & -1.2224 & -2.5168 & 0.4856 \\
\hline & & 90 & 1.00 & 3.0028 & 0.0000 & 1.4056 & -9.6093 & 0.0000 & -0.4668 & 0.0001 & -4.0195 & -0.0001 \\
\hline & 3 & 0 & 1.00 & 3.0385 & 0.0000 & 3.3770 & -9.1504 & 0.0000 & -1.6489 & 0.0000 & -5.3902 & 0.0001 \\
\hline & & 30 & 0.37 & 1.5389 & 0.0000 & -0.7162 & -4.5253 & -1.2157 & 0.0198 & -1.3950 & -2.1651 & 0.5424 \\
\hline & & 60 & 0.45 & 1.7527 & 0.0000 & -0.5745 & -5.3281 & -0.9351 & 0.1169 & -1.9093 & -2.0826 & 0.6442 \\
\hline & & 90 & 1.00 & 3.0125 & 0.0000 & 1.3065 & -10.0011 & 0.0000 & -0.2339 & 0.0000 & -3.3754 & -0.0002 \\
\hline & 4 & 0 & 1.00 & 3.0452 & 0.0000 & 4.0344 & -9.1363 & 0.0000 & -1.8378 & 0.0000 & -5.4781 & 0.0001 \\
\hline & & 30 & 0.31 & 1.3371 & 0.0000 & -1.1222 & -3.9665 & -1.3006 & -0.1054 & -1.5222 & -1.9104 & 0.4942 \\
\hline & & 60 & 0.41 & 1.6499 & -0.0001 & -0.7620 & -5.1556 & -1.0207 & -0.0906 & -2.4550 & -1.9034 & 0.6985 \\
\hline & & 90 & 1.00 & 3.0300 & 0.0000 & 1.2884 & -10.4269 & 0.0000 & -0.0851 & 0.0000 & -2.7909 & -0.0002 \\
\hline
\end{tabular}

\section{References}

1] M.D. Kuruppu, Y. Obara, M.R. Ayatollahi, K.P. Chong, T. Funatsu, ISRM-suggested method for determining the mode I static fracture toughness using semi-circular bend specimen, Rock Mech. Rock Eng. 47 (2014) 267-274.

[2] I. Lim, I. Johnston, S. Choi, J. Boland, Fracture testing of a soft rock with semicircular specimens under three-point bending. Part 1-mode I, Int. J. Rock Mech. Min. Sci. Geomech. Abstracts 31 (1994) 185-197.

[3] F. Dai, R. Chen, K. Xia, A semi-circular bend technique for determining dynamic fracture toughness, Exp. Mech. 50 (2010) 783-791.

[4] J. Akbardoost, M. Ayatollahi, M. Aliha, M. Pavier, D. Smith, Size-dependent fracture behavior of Guiting limestone under mixed mode loading, Int. J. Rock Mech. Min. Sci. 71 (2014) 369-380.

[5] F. Berto, M. Ayatollahi, T. Borsato, P. Ferro, Local strain energy density to predict size-dependent brittle fracture of cracked specimens under mixed mode loading, Theoret. Appl. Fract. Mech. 86 (2016) 217-224.

[6] M.D. Wei, F. Dai, N.W. Xu, T. Zhao, Y. Liu, An experimental and theoretical assessment of semi-circular bend specimens with chevron and straight-through notches for mode I fracture toughness testing of rocks, Int. J. Rock Mech. Min. Sci. 99 (2017) 28-38.

[7] A. Pakdaman, M. Moosavi, S. Mohammadi, Experimental and numerical investigation into the methods of determination of mode I static fracture toughness of rocks, Theoret. Appl. Fract. Mech. 100 (2019) 154-170.

[8] B. Bahrami, M.R. Ayatollahi, A.M. Mirzaei, M.Y. Yahya, Support type influence on rock fracture toughness measurement using semi-circular bending specimen, Rock Mech. Rock Eng. (2019).

[9] K.P. Chong, M.D. Kuruppu, J.S. Kuszmaul, Fracture toughness determination of layered materials, Eng. Fract. Mech. 28 (1987) 43-54.

[10] F. Dai, K. Xia, Laboratory measurements of the rate dependence of the fracture toughness anisotropy of Barre granite, Int. J. Rock Mech. Min. Sci. 60 (2013) 57-65.

[11] M. Kataoka, Y. Obara, M. Kuruppu, Estimation of fracture toughness of anisotropic rocks by semi-circular bend (SCB) Tests Under Water Vapor Pressure, Rock Mech. Rock Eng. 48 (2015) 1353-1367.

[12] H.P. Lee, J.E. Olson, J. Holder, J.F.W. Gale, R.D. Myers, The interaction of propagating opening mode fractures with preexisting discontinuities in shale, J. Geophys. Res.: Solid Earth 120 (2015) 169-181.

[13] H. Wang, F. Zhao, Z. Huang, Y. Yao, Experimental study of mode-I fracture toughness for layered shale based on two ISRM-suggested methods, Rock Mech. Rock Eng. (2017).

[14] J. Shang, Y. Gui, Z. Zhao, Broad-spectrum fracture toughness of an anisotropic sandstone under mixed-mode loading, Theoret. Appl. Fract. Mech. 96 (2018) $556-575$.

[15] N. Dutler, M. Nejati, B. Valley, F. Amann, G. Molinari, On the link between fracture toughness, tensile strength, and fracture process zone in anisotropic rocks, Eng. Fract. Mech. 201 (2018) 56-79.

[16] M. Nejati, A. Aminzadeh, F. Amann, M.O. Saar, T. Driesner, Mode I fracture growth in anisotropic rocks: Theory and experiment, Int. J. Solids Struct. 195 (2020) 74-90.

[17] I. Lim, I. Johnston, S. Choi, Stress intensity factors for semi-circular specimens under three-point bending, Eng. Fract. Mech. 44 (1993) 363-382.

[18] M. Ayatollahi, M. Aliha, Wide range data for crack tip parameters in two disc-type specimens under mixed mode loading, Comput. Mater. Sci. 38 (2007) 660-670.

[19] M. Nejati, A. Aminzadeh, M.O. Saar, T. Driesner, Modified semi-circular bend test to determine the fracture toughness of anisotropic rocks, Eng. Fract. Mech. 213 (2019) 153-171.

[20] M. Nejati, A. Aminzadeh, T. Driesner, M.O. Saar, On the directional dependency of Mode I fracture toughness in anisotropic rocks, Theoret. Appl. Fract. Mech. 107 (2020) 102494.

[21] M.D. Wei, F. Dai, N.W. Xu, T. Zhao, Stress intensity factors and fracture process zones of ISRM-suggested chevron notched specimens for mode I fracture toughness testing of rocks, Eng. Fract. Mech. 168 (2016) 174-189.

[22] M.D. Wei, F. Dai, N.W. Xu, Y. Liu, T. Zhao, Fracture prediction of rocks under mode I and mode II loading using the generalized maximum tangential strain criterion,
Eng. Fract. Mech, 186 (2017) 21-38.

[23] M.D. Wei, F. Dai, Y. Liu, N.W. Xu, T. Zhao, An experimental and theoretical comparison of CCNBD and CCNSCB specimens for determining mode I fracture toughness of rocks, Fatigue Fract. Eng. Mater. Struct. 41 (2018) 1002-1018.

[24] B.L. Karihaloo, H. Abdalla, Q.Z. Xiao, Coefficients of the crack tip asymptotic field for a standard compact tension specimen, Int. J. Fract. 118 (2002) 1-15.

[25] B.L. Karihaloo, Q.Z. Xiao, Higher order terms of the crack tip asymtotic field for a notched three-point bend beam, Int. J. Fract. 112 (2001) 111-128.

[26] Q.Z. Xiao, B.L. Karihaloo, Direct evaluation of accurate coefficients of the linear elastic crack tip asymptotic field, Fatigue Fract. Eng. Mater. Struct. 26 (2003) 719-729.

[27] B.L. Karihaloo, H. Abdalla, Q.Z. Xiao, Coefficients of the crack tip asymptotic field for wedge splitting specimens, Eng. Fract. Mech. 70 (2003) 2407-2420.

[28] B. Karihaloo, H. Abdalla, Q. Xiao, Size effect in concrete, Eng. Fract. Mech. 70 (2003) 979-993.

[29] B.L. Karihaloo, H.M. Abdalla, Q.Z. Xiao, Deterministic size effect in the strength of cracked concrete structures, Cem. Concr. Res. 36 (2006) 171-188.

[30] M.D. Wei, F. Dai, J.W. Zhou, Y. Liu, J. Luo, A Further Improved Maximum Tangential Stress Criterion for Assessing Mode I Fracture of Rocks Considering Nonsingular Stress Terms of the Williams Expansion, Rock Mech. Rock Eng. 51 (2018) 3471-3488.

[31] M.R. Ayatollahi, M. Nejati, S. Ghouli, The finite element over-deterministic method to calculate the coefficients of crack tip asymptotic fields in anisotropic planes, Eng. Fract. Mech. 231 (2020) 106982.

[32] S.G. Lekhnitskii, Anisotropic Plates, Gordon and Breach Science Publishers, New York, USA, 1968.

[33] B. Saint Venant, Sur la distributiondes élasticités autour de chaque point d'un solide ou d'unmilieu de contexture quelconque, particulièrement lorsqu'il est amorphe sans être isotrope, Journal de Math. Pures et Appliquées VIII (1863) 257-430.

[34] A. Aminzadeh, A. Fahimifar, M. Nejati, On brazilian disk test for mixed-mode i/ii fracture toughness experiments of anisotropic rocks, Theoret. Appl. Fract. Mech. 102 (2019) 222-238.

[35] S. Ghouli, M.R. Ayatollahi, M. Nejati, On higher order parameters in cracked composite plates under far-field pure shear, Fatigue Fract. Eng. Mater. Struct. 43 (2020) 568-585.

[36] M. Ayatollahi, M. Zakeri, An improved definition for mode I and mode II crack problems, Eng. Fract. Mech. 175 (2017) 235-246.

[37] B. Bahrami, M.R. Ayatollahi, I. Sedighi, M. Yazid Yahya, An insight into mode II fracture toughness testing using SCB specimen, Fatigue Fract. Eng. Mater. Struct. 42 (2019) 1991-1999.

[38] I. Sedighi, M.R. Ayatollahi, B. Bahrami, A statistical approach on the support type effect on mode I fracture toughness determined using semi-circular bend (SCB) specimen, Eng. Fract. Mech. 226 (2020) 106891.

[39] R.S. Barsoum, On the use of isoparametric finite elements in linear fracture mechanics, Int. J. Numer. Meth. Eng. 10 (1976) 25-37.

[40] M. Nejati, A. Paluszny, R.W. Zimmerman, On the use of quarter-point tetrahedral finite elements in linear elastic fracture mechanics, Eng. Fract. Mech. 144 (2015) 194-221.

[41] M. Ayatollahi, J. Akbardoost, Size effects on fracture toughness of quasi-brittle materials - A new approach, Eng. Fract. Mech. 92 (2012) 89-100.

[42] J. Akbardoost, M. Ayatollahi, Experimental analysis of mixed mode crack propa gation in brittle rocks: The effect of non-singular terms, Eng. Fract. Mech. 129 (2014) 77-89.

[43] F. Erdogan, G.C. Sih, On the Crack Extension in Plates Under Plane Loading and Transverse Shear, J. Basic Eng. 85 (1963) 519.

[44] G.C. Sih, Strain-energy-density factor applied to mixed mode crack problems, Int. J. Fract. 10 (1974) 305-321.

[45] Kaung Jain Chang, On the maximum strain criterion-a new approach to the angled crack problem, Eng. Fract. Mech. 14 (1981) 107-124.

[46] V.E. Saouma, M.L. Ayari, D.A. Leavell, Mixed mode crack propagation in homogeneous anisotropic solids, Eng. Fract. Mech. 27 (1987) 171-184.

[47] Z. Ye, M. Ayari, Prediction of crack propagation in anisotropic solids, Eng. Fract. Mech. 49 (1994) 797-808.

[48] M.L. Ayari, Z. Ye, Maximum strain theory for mixed mode crack propagation in 
anisotropic solids, Eng. Fract. Mech. 52 (1995) 389-400.

[49] M. Ryvkin, K-Dominance zone for a semi-infinite mode I crack in a sandwich composite, Int. J. Solids Struct. 37 (2000) 4825-4840.

[50] D.J. Shim, G.H. Paulino, R.H. Dodds, A boundary layer framework considering material gradation effects, Eng. Fract. Mech. 73 (2006) 593-615.

[51] C.T. Sun, H. Qian, Brittle fracture beyond the stress intensity factor, J. Mech. Mater. Struct. 4 (2009) 743-753.

[52] B. Kumar, S. Chitsiriphanit, C. Sun, Significance of K-dominance zone size and nonsingular stress field in brittle fracture, Eng. Fract. Mech. 78 (2011) 2042-2051.

[53] A. Nazmus Sakib, A. Adnan, On the size-dependent critical stress intensity factor of confined brittle nanofilms, Eng. Fract. Mech. 86 (2012) 13-22.

[54] S.H. Cheng, C.T. Sun, Size-dependent fracture toughness of nanoscale structures: crack-tip stress approach in molecular dynamics, J. Nanomech. Micromech. 4
(2014) A4014001-1-A4014001-8.

[55] D. Touliatou, M.A. Wheel, K-dominance and size effect in mode I fracture of brittle materials with low to medium porosity, Eng. Fract. Mech. 201 (2018) 269-281.

[56] M. Kuna, Finite Elements in Fracture Mechanics. volume 201 of Solid Mechanics and Its Applications. Springer, Netherlands, Dordrecht, 2013.

[57] M. Wei, F. Dai, N. Xu, T. Zhao, Experimental and numerical investigation of cracked chevron notched Brazilian disc specimen for fracture toughness testing of rock, Fatigue Fract. Eng. Mater. Struct. 41 (2018) 197-211.

[58] R. Nath Singh, G. Sun, A numerical and experimental investigation for determining fracture toughness of Welsh limestone, Min. Sci. Technol. 10 (1990) 61-70.

[59] M. Wei, F. Dai, N. Xu, T. Zhao, K. Xia, Experimental and numerical study on the fracture process zone and fracture toughness determination for ISRM-suggested semi-circular bend rock specimen, Eng. Fract. Mech. 154 (2016) 43-56. 